\title{
Stability Properties of the Riemann Ellipsoids
}

\author{
Francesco Fassò* $\quad$ Debra Lewis ${ }^{\dagger}$
}

(January 31, 2000)

\begin{abstract}
We study the ellipticity and the "Nekhoroshev stability" (stability properties for finite, but very long, time scales) of the Riemann ellipsoids. We provide numerical evidence that the regions of ellipticity of the ellipsoids of types II and III are larger than those found by Chandrasekhar in the 60's and that all Riemann ellipsoids, except a finite number of codimension one subfamilies, are Nekhoroshev-stable. We base our analysis on a Hamiltonian formulation of the problem on a covering space, using recent results from Hamiltonian perturbation theory.
\end{abstract}

\section{Introduction}

A. The Dirichlet problem consists of the "linear" motions of an ideal, incompressible, homogeneous, self-gravitating fluid mass having an ellipsoidal shape at any instant. This problem originated in attempts to determine the shapes of the planets, and has a long and distinguished history (see e.g. chapter 1 of [6] and references therein). Let us only remark here that Newton and Maclaurin established the existence of rigidly rotating oblate spheroids and that Jacobi described rigidly rotating asymmetric ellipsoids. Dirichlet [9] proved the existence of "linear" fluid motions, such that the position $x(t, y)$ at time $t$ of any material particle $y$ is given by

$$
x(t, y)=F(t) y, \quad F(t) \in \mathrm{SL}(3) .
$$

If, as we assume, the reference configuration of the fluid mass is a ball of radius $\rho$, then the free surface of the fluid determined by the motion (1.1) is at every instant an ellipsoid with semiaxes

\footnotetext{
${ }^{*}$ Università di Padova, Dipartimento di Matematica Pura e Applicata, Via G. Belzoni 7, 35131 Padova, Italy. (E-mail: fasso@math.unipd.it). Partially supported by a CNR-NATO Fellowship, by DOE contract DEFG0395ER25245-A000 while visiting the Mathematics Department, University of California at Santa Cruz, and by MURST protocol 9802261238_008.

${ }^{\dagger}$ Mathematics Department, University of California, Santa Cruz, CA 95064, USA. (E-mail: lewis@math.ucsc.edu). Partially supported by NSF grant DMS-9802378 and the Santa Fe Institute.
} 
$\rho a_{1}, \rho a_{2}, \rho a_{3}$, where $a_{1}, a_{2}$ and $a_{3}$ are the singular values of the matrix $F$ (namely, the eigenvalues of $\sqrt{F F^{T}}$.

The Maclaurin spheroids and Jacobi ellipsoids are steady rigid motions of the Dirichlet problem. Dedekind identified a new class of steady, but non-rigid, motions of the problem; they were constructed by transposition of the Jacobi ellipsoids, thus bringing the particle relabelling symmetry of the system into play. Riemann reformulated the equations of motion of the Dirichlet problem in a particularly convenient form for studying asymmetric configurations (as we shall show, this reformulation corresponds to the passage to a four-to-one covering space). Riemann also classified all steady motions of the system, showing that they are generated by combinations of spatial and body symmetries, and studied their Lyapunov (or nonlinear) orbital stability.

The steady motions found by Riemann, which are called Riemann ellipsoids, are motions (1.1) such that

$$
F(t)=\exp \left(t \Omega_{l}\right) A \exp \left(-t \Omega_{r}\right)
$$

for some constant diagonal matrix $A=\operatorname{diag}\left(a_{1}, a_{2}, a_{3}\right)$ and constant antisymmetric matrices $\Omega_{l}$ and $\Omega_{r}$. Except for the special case of the Jacobi ellipsoids, for which $\Omega_{r}=0$, Riemann ellipsoids do not perform rigid motions: the motion is the composition of an internal rotation, a stretch along the principal axes, and a spatial rotation; the free surface retains a rotating ellipsoidal shape, but the fluid particles describe rosette-shaped motions which are either closed (periodic) or open (quasiperiodic) depending on the two angular frequencies $\omega_{l}$ and $\omega_{r}$ (that is, the vectors corresponding to the antisymmetric matrices $\Omega_{l}$ and $\Omega_{r}$ ). Riemann proved that there are five types of Riemann ellipsoids with distinct semiaxes, characterized by different relations between the angular velocities and the semiaxes. For two types (hereafter referred to as $\mathrm{S}_{2}$ and $\mathrm{S}_{3}$ ), both $\omega_{l}$ and $\omega_{r}$ are parallel to the same principal axis of the ellipsoid; this axis is either the shortest $\left(\mathrm{S}_{3}\right)$ or the middle $\left(\mathrm{S}_{2}\right)$ one. For the other three types, called I, II, and III, both $\omega_{l}$ and $\omega_{r}$ belong to one of the two principal planes containing the longest axis of the ellipsoid. I

Since the Riemann ellipsoids are reduced equilibria, namely equilibria modulo symmetries, of the Dirichlet problem, it is meaningful to study their stability modulo symmetries, which we will frequently refer to simply as stability. Riemann found that all ellipsoids of type $\mathrm{S}_{3}$ and a subfamily of the $\mathrm{S}_{2}$ ellipsoids are stable. Riemann's analysis does not lead to any definite conclusion about the (in)stability of the remaining ellipsoids, because they are saddle points of his Lyapunov function.

More recently, Chandrasekhar studied the 'ellipticity', or 'spectral stability', of the Riemann ellipsoids. We say here that a (relative) equilibrium is elliptic if the eigenvalues of the linearization at the equilibrium are purely imaginary; note that, accordingly, we treat the case of zero eigenvalues as elliptic, even though this is not customary. Chandrasekhar [4] [5] [6] numerically found a region of ellipticity of the ellipsoids of type $\mathrm{S}_{2}$ larger than the region of Lyapunov stability found by Riemann. He also found nonempty regions of elliptic ellipsoids of type I and III, but none of type II. As we shall see, some of these conclusions are not entirely correct, and in fact require substantial modification.

B. For a Hamiltonian system such as the Dirichlet problem, ellipticity is a necessary, but not sufficient, condition for Lyapunov stability. Therefore, the ellipticity analysis is of twofold interest: on one hand, the absence of ellipticity implies Lyapunov instability; on the other hand, it opens up the problem of the stability properties of those Riemann ellipsoids that are elliptic, but of

\footnotetext{
${ }^{1}$ The distinction between $\mathrm{S}_{2}$ and $\mathrm{S}_{3}$ ellipsoids that we make here is not standard; Chandrasekhar calls them collectively ellipsoids of type S.
} 
undetermined Lyapunov stability. The present article is mainly devoted to a study of the latter question.

First of all, as a prerequisite for this analysis, we repeat the Lyapunov stability and the ellipticity analysis of the Riemann ellipsoids. While our analysis confirms Riemann's Lyapunov stability results, we find significant discrepancies with Chandrasekhar's results: there is a nonempty region of elliptic ellipsoids of type II and a region of elliptic ellipsoids of type III that is substantially larger than the one reported by Chandrasekhar. Therefore, the regions of known (Lyapunov) instability of the ellipsoids of types II and III are substantially smaller than those found by Chandrasekhar. Moreover, the ellipticity regions of the ellipsoids of type I, II and III have a finer and more complex structure than was previously realized.

The main goal of the article is to use the methods of Hamiltonian perturbation theory to investigate the long-time stability properties of the elliptic Riemann ellipsoids of undetermined Lyapunov stability. In fact, the Dirichlet problem is a Hamiltonian system with symmetry and the Riemann ellipsoids are the relative equilibria of this system. They correspond to the equilibria of the reduced system, which has four degrees of freedom for generic values of the momenta and three degrees of freedom in special cases. (The reduced phase space is in fact an orbifold, with a singular set containing the Riemann ellipsoids of type $\mathrm{S}$, but this difficulty is overcome by passing to Riemann's covering space.) Since the reduced system has more than two degrees of freedom, KAM theory cannot be used to show the stability of its equilibria. Therefore, we shall base our investigation on Nekhoroshev theory, which allows one to obtain "practical" stability results, namely, to prove that motions starting sufficiently near the equilibrium remain near it for times growing exponentially with (some power) of the initial distance; specifically, we shall use some recent results obtained in [2] (see also [10], [17], [11]). The results of our investigation indicate that all elliptic Riemann ellipsoids are Nekhoroshev-stable, with the possible exception of a finite number of codimension one families of ellipsoids satisfying certain low-order resonance conditions.

In view of the complexity of the system, we resort to numerical methods at certain steps in the ellipticity analysis and in the construction of the normal forms for the reduced Hamiltonian. We numerically compute the eigenvalues of the linearization and numerically construct the normal forms for a finite (though rather large and, we think, significant) number of values of the momentum. Therefore, the forementioned results are not rigorous, but we think that our analysis provides strong evidence for our conclusions.

C. Our analysis is based on a Hamiltonian formulation (on a covering manifold) of the Dirichlet problem, which is briefly developed in Section 2. For the sake of brevity, we skip most of the computational details and focus on the conceptual features. The Riemann ellipsoids are described in Section 3. Overall, Sections 2 and 3 contain a treatment of the Dirichlet problem which, although concise, is complete and detailed; this seems necessary, because the existing literature does not seem to include a comparably comprehensive treatment.

Sections 4-6 contain the ellipticity and the Nekhoroshev-stability analyses. In Section 4 we review the results on Lyapunov stability and instability and describe the results of our ellipticity analysis; a detailed comparison with Chandrasekhar's results is included in Appendix B. We briefly review the necessary notions for the Nekhoroshev-stability analysis in Section 5 and describe the results for the Riemann ellipsoids in Section 6. A Conclusion follows.

All of the numerical computations have been performed using the software package Mathematica. 


\section{The Dirichlet Problem}

A. Dirichlet's equations. Dirichlet [9] showed that the linear motions (1.1) form an invariant submanifold of the phase space of an ideal, incompressible, homogeneous, self-gravitating fluid, with the boundary condition that the pressure is constant at the free surface. He expressed the restriction of the hydrodynamic equations of motion to this submanifold as equations for the curve $F(t) \in \mathrm{SL}(3)$, obtaining a second order system on SL(3).

To describe these results, we introduce the following notation, which will be used throughout the article. We denote by $\cdot$ and \|\| the Euclidean scalar product and norm on $\mathbb{R}^{3}$ and by $D_{\rho}$ the ball $\left\{y \in \mathbb{R}^{3}:\|y\| \leq \rho\right\}$. We use the standard inner product and norm on $L(3)$ given by

$$
\langle A, B\rangle=\operatorname{tr}\left(A B^{T}\right) \text { and }|A|^{2}=\langle A, A\rangle .
$$

(Note that the subspaces of symmetric and antisymmetric matrices are $\langle$,$\rangle -orthogonal comple-$ ments of one another in $L(3)$.) We shall frequently identify $\mathbb{R}^{3}$ and so(3) by means of the isomorphism $\omega \mapsto \hat{\omega}$, where $\hat{\omega} \xi=\omega \times \xi$ for all $\xi \in \mathbb{R}^{3}$. Note that $\langle\hat{\omega}, \hat{\xi}\rangle=2 \omega \cdot \xi$ for all $\omega, \xi \in \mathbb{R}^{3}$. Following Riemann, we utilize the "singular value decomposition" of matrices, nowadays a standard tool in numerical linear algebra: If $F \in \mathrm{SL}(3)$ and $a_{1}, a_{2}, a_{3}$ are its singular values, then there exist matrices $U_{l}, U_{r} \in \mathrm{SO}(3)$ such that

$$
F=U_{l} A U_{r}^{T}, \quad A=\operatorname{diag}\left(a_{1}, a_{2}, a_{3}\right) .
$$

We shall refer to the triplet $\left(A, U_{l}, U_{r}\right)$ as a "SVD" of the matrix $F$. The matrices $A, U_{l}$ and $U_{r}$ are not unique, but one can clearly fix $A$ by ordering its entries, e.g. $a_{1} \geq a_{2} \geq a_{3}$ (see also Proposition 11 below). Finally, we set

$$
\mathcal{V}(a)=-2 \pi g \int_{0}^{\infty}\left[\left(s+a_{1}^{2}\right)\left(s+a_{2}^{2}\right)\left(s+a_{3}^{2}\right)\right]^{-1 / 2} d s,
$$

where $g$ denotes the gravitational constant; by a slight abuse of notation, we shall regard $\mathcal{V}$ and its derivatives as functions either of $a=\left(a_{1}, a_{2}, a_{3}\right)$ or of the diagonal matrix $A=\operatorname{diag}\left(a_{1}, a_{2}, a_{3}\right)$.

Dirichlet's result can now be expressed as follows: Consider a curve $t \mapsto F(t) \subset \mathrm{SL}(3)$ defined on some open interval $\mathcal{J} \subset \mathbb{R}$ and let $\left(A(t), U_{l}(t), U_{r}(t)\right)$ be any singular value decomposition of $F(t)$. Then the map

$$
x: \mathcal{J} \times D_{\rho} \rightarrow \mathbb{R}^{3}, \quad(t, y) \mapsto F(t) y
$$

is a solution of the hydrodynamical equation for an ideal, incompressible, homogeneous, selfgravitating fluid with constant pressure at the boundary iff

$$
\mathbb{P}_{F}\left[\ddot{F}+U_{l} \mathcal{V}^{\prime}(A) U_{r}^{T}\right]=0,
$$

where $\mathcal{V}^{\prime}=\operatorname{diag}\left(\frac{\partial \mathcal{V}}{\partial a_{1}}, \frac{\partial \mathcal{V}}{\partial a_{2}}, \frac{\partial \mathcal{V}}{\partial a_{3}}\right)$ and $\mathbb{P}_{F} G=G-\frac{1}{3}\langle G, F\rangle F^{-T}$ for any $G \in L(3) . \quad$ Note that the matrix $U_{l} \mathcal{V}^{\prime}(A) U_{r}^{T}$ is independent of the particular SVD of $F$ used; this is proven by observing that, if $U, V \in \mathrm{SO}(3)$ and $A=U A^{\prime} V$ with $A$ and $A^{\prime}$ diagonal, then $\mathcal{V}^{\prime}(A)=\mathcal{V}^{\prime}\left(U A^{\prime} V\right)=U \mathcal{V}^{\prime}\left(A^{\prime}\right) V$. In fact, Dirichlet used the polar decomposition, not the SVD, when writing the force term in the equations of motion.

Remark: In his work on Dirichlet problem, Chandrasekhar required that the relative pressure $p-p_{o}$, where $p_{o}$ is the constant pressure on the surface of the fluid, be nonnegative at any point 
of the fluid. Following Riemann, we do not make this assumption. (There are many fluids that support even very high tensile states.)

Since the gravitational forces are conservative and invariant under spatial rotations and particle relabelling, equations (2.2) inherit the conservation of energy, angular momentum and circulation from the hydrodynamic equations. For future reference, we observe that if we set $c=\frac{m \rho^{2}}{5}$, where $m$ denotes the mass of the fluid, then the energy of any motion (1.1) is $c\left[\frac{1}{2}|\dot{F}|^{2}+\mathcal{V}(a)\right]$ and the angular momentum is the vector $k$ satisfying $\hat{k}=2 c \operatorname{skew}\left(\dot{F} F^{T}\right)$; here $\operatorname{skew}(G)=\frac{1}{2}\left(G-G^{T}\right)$. The conservation of circulation is equivalent to the constancy of $\operatorname{skew}\left(F^{T} \dot{F}\right)$.

B. Riemann's equation. Shortly after Dirichlet's work appeared, Riemann 20] rewrote equation (2.2) using what is nowadays called the singular value decomposition of matrices and left-trivializing the factors $T \mathrm{SO}(3)$, namely expressing the tangent vectors $\dot{U}_{l}$ and $\dot{U}_{r}$ in terms of the antisymmetric matrices $\Omega_{l}=U_{l}^{T} \dot{U}_{l}$ and $\Omega_{r}=U_{r}^{T} \dot{U}_{r}$. Once these substitutions have been made, equation (2.2) takes the form

$$
\mathbb{P}_{A}\left[\ddot{A}+2\left(\Omega_{l} \dot{A}-\dot{A} \Omega_{r}\right)+\left(\dot{\Omega}_{l} A-A \dot{\Omega}_{r}\right)+\left(\Omega_{l}^{2} A-2 \Omega_{l} A \Omega_{r}+A \Omega_{r}^{2}\right)+\mathcal{V}^{\prime}(A)\right]=0
$$

(which should be compared with equations $(\alpha)$ in [20]). This equation determines a second order system on $\mathcal{A} \times \mathrm{SO}(3) \times \mathrm{SO}(3)$, where

$$
\mathcal{A}=\left\{\operatorname{diag}\left(a_{1}, a_{2},\left(a_{1} a_{2}\right)^{-1}\right): a_{1}>a_{2}>a_{1}^{-1 / 2}\right\}
$$

(if $A$ has repeated eigenvalues, $\dot{\Omega}_{l}$ and $\dot{\Omega}_{r}$ are not uniquely determined by (2.3)). This system is equivalent to the restriction of Dirichlet's equation (2.2) to the submanifold

$$
Q=\{F \in \mathrm{SL}(3): F \text { has distinct singular values }\} \text {. }
$$

More precisely, because of the nonuniqueness of the SVD, the two systems are related not by a diffeomorphism, but by a four-to-one covering map:

Proposition 1 The map

$$
\mathcal{C}: \mathcal{A} \times \mathrm{SO}(3) \times \mathrm{SO}(3) \rightarrow Q, \quad\left(A, U_{l}, U_{r}\right) \mapsto U_{l} A U_{r}^{T}
$$

is a 4-fold covering map which maps solutions of equation (2.9) into solutions of equation (2.9).

Proof. If $\left(A, U_{l}, U_{r}\right)$ and $\left(A, V_{l}, V_{r}\right)$ are any two SVDs of $F \in Q$, with $A=\operatorname{diag}\left(a_{1}, a_{2}, a_{3}\right)$, $a_{1}>a_{2}>a_{3}$, then $V_{l}=R_{i} U_{l}$ and $V_{r}=R_{i} U_{r}$ for some $i=0, \ldots, 3$, where

$$
R_{0}=\mathbf{1}, \quad R_{1}=\operatorname{diag}(1,-1,-1), \quad R_{2}=\operatorname{diag}(-1,1,-1), \quad \text { and } \quad R_{3}=\operatorname{diag}(-1,-1,1) .
$$

Thus, the map $\mathcal{C}$ is four-to-one. That it is a submersion is proven, for instance, by showing that the determinant of its Jacobian is nonzero. (Using $a_{1}, a_{2}$ as coordinates on $\mathcal{A}$ and left-trivializing the factors $\operatorname{TSO}(3)$, one obtains the determinant $\left(a_{1}^{2}-a_{2}^{2}\right)\left(a_{1}^{2}-a_{3}^{2}\right)\left(a_{2}^{2}-a_{3}^{2}\right)$.)

In Riemann's formulation, the use of the SVD has the advantage that the absence of the matrices $U_{l}$ and $U_{r}$ from the equation (2.3) makes manifest the $\mathrm{SO}(3) \times \mathrm{SO}(3)$ invariance of the Dirichlet 
problem. From our perspective, the formulation on the covering also has the advantage that, once we pass to the Hamiltonian formulation, the symplectically reduced phase space associated to the cotangent bundle of the covering is a manifold, while reduction of the system on $T^{*} Q$ produces singularities.

Remarks: (i) The submanifold $Q$ is not invariant under the dynamics of the Dirichlet problem (axisymmetric configurations can evolve into asymmetric ones, and vice versa) but this is not a problem for our analysis: the Riemann ellipsoids with asymmetric configurations clearly belong to $Q$ for all time and, since $Q$ is open, it will be a consequence of our stability estimates that nearby motions also remain in $Q$ for the time under consideration.

(ii) The restriction to the case of distinct singular values excludes all axisymmetric Riemann ellipsoids. The stability properties of the Maclaurin spheroids, which rotate rigidly about the axis of symmetry, are completely known (see [20][6] [14]); the remaining axisymmetric ellipsoids merit further investigation.

C. Hamiltonian formulation. We now describe the Hamiltonian formulation of Riemann's equation (2.3) on the cotangent bundle of the covering manifold $\mathcal{A} \times \mathrm{SO}(3) \times \mathrm{SO}(3)$. We shall use the first two diagonal entries $a_{1}$ and $a_{2}$ as coordinates on the set $\mathcal{A}$, by means of the diffeomorphism $\alpha: \mathcal{B} \rightarrow \mathcal{A}$, where

$$
\mathcal{B}=\left\{\left(b_{1}, b_{2}\right) \in \mathbb{R}^{2}: b_{1}>b_{2}>b_{1}^{-1 / 2}\right\} \quad \text { and } \quad \alpha\left(b_{1}, b_{2}\right):=\operatorname{diag}\left(b_{1}, b_{2}, \frac{1}{b_{1} b_{2}}\right) .
$$

(We denote by $b=\left(b_{1}, b_{2}\right)$ the first two singular values, so as to distinguish $b \in \mathbb{R}^{2}$ from $a \in \mathbb{R}^{3}$; wherever we write $b_{3}$, it stands for $\left(b_{1} b_{2}\right)^{-1}$, not for an independent variable.) Furthermore we identify the cotangent bundle of each factor $\mathrm{SO}(3)$ with $\mathrm{SO}(3) \times \mathbb{R}^{3}$ as follows: we first identify the cotangent space $T_{U}^{*} \mathrm{SO}(3)$ with $T_{U} \mathrm{SO}(3)$ by means of the inner product $\frac{1}{2}\langle$,$\rangle (see (2.1)) and then$ identify $T_{U} \mathrm{SO}(3)$ with $\mathbb{R}^{3}$ by means of the ${ }^{\wedge}$ isomorphism, thus associating to each $\mu \in T_{U}^{*} \mathrm{SO}(3)$ the unique $m \in \mathbb{R}^{3}$ satisfying

$$
\mu \cdot U \hat{\omega}=\frac{1}{2}\langle\hat{m}, \hat{\omega}\rangle=m \cdot \omega \quad \forall \omega \in \mathbb{R}^{3} .
$$

After these identifications, we work on the sixteen-dimensional manifold

$$
\mathcal{M}=\mathcal{B} \times \mathbb{R}^{2} \times(\mathrm{SO}(3))^{2} \times\left(\mathbb{R}^{3}\right)^{2},
$$

which is diffeomorphic to $T^{*}(\mathcal{A} \times \mathrm{SO}(3) \times \mathrm{SO}(3))$. We shall denote by $(b, c, U, m)$ the elements of $\mathcal{M}$, with $b \in \mathcal{B}, c \in \mathbb{R}^{2}, U=\left(U_{l}, U_{r}\right) \in\left(\mathrm{SO}(3)^{2}\right)$, and $m=\left(m_{l}, m_{r}\right) \in\left(\mathbb{R}^{3}\right)^{2}$ and use the induced scalar and vector products on $\left(\mathbb{R}^{3}\right)^{2}$ given by

$$
\left(\omega_{l}, \omega_{r}\right) \cdot\left(\xi_{l}, \xi_{r}\right)=\omega_{l} \cdot \xi_{l}+\omega_{r} \cdot \xi_{r}, \quad\left(\omega_{l}, \omega_{r}\right) \times\left(\xi_{l}, \xi_{r}\right)=\left(\omega_{l} \times \xi_{l}, \omega_{r} \times \xi_{r}\right) .
$$

When writing tangent vectors to $\mathcal{M}$ we shall again left-trivialize and identify the tangent spaces to the two factors $\mathrm{SO}(3)$ with $\mathbb{R}^{3}$; thus, $(v, w, \omega, n)$ denotes the vector $\left(v, w,\left(U_{l} \hat{\omega}_{l}, U_{r} \hat{\omega}_{r}\right), n\right) \in$ $T_{(b, c, U, m)} \mathcal{M}$. Finally, in order to avoid the introduction of a new symbol, we write $\mathcal{V}(b)$ to denote $\mathcal{V}\left(b_{1}, b_{2}, \frac{1}{b_{1} b_{2}}\right)$. 
Proposition 2 Riemann's equation (2.3) on $\mathcal{A} \times \mathrm{SO}(3) \times \mathrm{SO}(3)$ is equivalent to the Hamiltonian system on the manifold $\mathcal{M}$ defined by the Hamiltonian

$$
H(b, c, U, m)=\frac{1}{2} c \cdot \mathbb{K}(b) c+\frac{1}{2} m \cdot \mathbb{J}(b) m+\mathcal{V}(b),
$$

where

$$
\begin{aligned}
\mathbb{K}(b) & =\frac{1}{b_{2}^{2} b_{3}^{2}+b_{1}^{2} b_{3}^{2}+b_{1}^{2} b_{2}^{2}}\left(\begin{array}{cc}
b_{1}^{2}\left(b_{2}^{2}+b_{3}^{2}\right) & -b_{3} \\
-b_{3} & b_{2}^{2}\left(b_{1}^{2}+b_{3}^{2}\right)
\end{array}\right) \\
\mathbb{J}(b) & =\left(\begin{array}{ll}
J_{1}(b) & J_{2}(b) \\
J_{2}(b) & J_{1}(b)
\end{array}\right) \quad \text { with }\left\{\begin{array}{l}
J_{1}(b)=\operatorname{diag}\left(\frac{b_{2}^{2}+b_{3}^{2}}{\left(b_{2}^{2}-b_{3}^{2}\right)^{2}}, \frac{b_{1}^{2}+b_{3}^{2}}{\left(b_{1}^{2}-b_{3}^{2}\right)^{2}}, \frac{b_{1}^{2}+b_{2}^{2}}{\left(b_{1}^{2}-b_{2}^{2}\right)^{2}}\right) \\
J_{2}(b)=\operatorname{diag}\left(\frac{2 b_{2} b_{3}}{\left(b_{2}^{2}-b_{3}^{2}\right)^{2}}, \frac{2 b_{1} b_{3}}{\left(b_{1}^{2}-b_{3}^{2}\right)^{2}}, \frac{2 b_{1} b_{2}}{\left(b_{1}^{2}-b_{2}^{2}\right)^{2}}\right),
\end{array}\right.
\end{aligned}
$$

and the "left-trivialized" (in the sense just specified) symplectic structure $\sigma$ is defined by

$$
\sigma(b, c, U, m)\left((v, w, \omega, n),\left(v^{\prime}, w^{\prime}, \omega^{\prime}, n^{\prime}\right)\right)=v \cdot w^{\prime}-v^{\prime} \cdot w+\omega \cdot n^{\prime}-n \cdot \omega^{\prime}+m \cdot\left(\omega \times \omega^{\prime}\right) .
$$

This proposition can be proven by a direct computation showing that Hamilton's equations for the Hamiltonian system defined by (2.5) and (2.6) are equivalent to Riemann's equations. Alternatively, one can observe that Riemann's equations are equivalent to the Lagrangian system defined on $T \mathcal{A} \times T \mathrm{SO}(3) \times T \mathrm{SO}(3)$ by the Lagrangian

$$
\mathcal{L}\left((A, \dot{A}), U_{l} \hat{\omega}_{l}, U_{r} \hat{\omega}_{r}\right)=\frac{1}{2}\left|\dot{A}+\hat{\omega}_{l} A-A \hat{\omega}_{r}\right|^{2}-\mathcal{V}(A)
$$

and construct the Hamiltonian system using the Legendre transform. For the sake of brevity, we skip all details, remarking only that the relation between 'angular momenta' and 'angular velocities' is $\omega=\mathbb{J}(b) m$.

Remark: The Lagrangian nature of Dirichlet's problem, with Lagrangian equal to the difference of the kinetic and the potential energies, is implicit in Riemann's work and has been repeatedly used since then. One can verify by a direct computation that the Euler-Lagrange equations for the Lagrangian (2.7) are equivalent to Riemann's equations (2.3).

The Hamiltonian system $(\mathcal{M}, H, \sigma)$ of Proposition 2 is invariant under the symplectic action $\Phi$ of $\mathrm{SO}(3) \times \mathrm{SO}(3)$ on $\mathcal{M}$ given by

$$
\Phi_{\left(R_{l}, R_{r}\right)}\left(b, c,\left(U_{l}, U_{r}\right), m\right)=\left(b, c,\left(R_{l} U_{l}, R_{r} U_{r}\right), m\right) .
$$

Since the group does not act on the factor $\mathcal{B} \times \mathbb{R}^{2}$, while it acts on each factor $\mathrm{SO}(3) \times \mathbb{R}^{3}$ as the left-trivialized cotangent lift of the left action of $\mathrm{SO}(3)$ on itself, all results from the elementary case of the left action of $\mathrm{SO}(3)$ on $T^{*} \mathrm{SO}(3)$ apply (see, e.g., [15] or [7]). Thus, one concludes that the action $\Phi$ has the momentum map

$$
\mathbf{J}: \mathcal{M} \rightarrow\left(\mathbb{R}^{3}\right)^{2}, \quad(a, c, U, m) \mapsto\left(U_{l} m_{l}, U_{r} m_{r}\right) .
$$

For any $\eta \in\left(\mathbb{R}^{3}\right)^{2}$, one may identify $\mathbf{J}^{-1}(\eta)$ and $\mathcal{B} \times \mathbb{R}^{2} \times(\mathrm{SO}(3))^{2}$, in which case the immersion $i_{\eta}: \mathbf{J}^{-1}(\eta) \rightarrow \mathcal{M}$ is given by $i_{\eta}(a, c, U)=\left(a, c, U,\left(U_{l}^{T} \eta_{l}, U_{r}^{T} \eta_{r}\right)\right)$. The two components $U_{l} m_{l}$ and $U_{r} m_{r}$ of the momentum map coincide, up to constant rescalings, with the total angular momentum and the circulation; see Section 2.A. 
The next Proposition, which describes the reduced system $\left(P_{\eta}, \sigma_{\eta}, H_{\eta}\right)$, also follows immediately from standard results for the reduction of an $S O(3)$ invariant Hamiltonian system on $T^{*} \mathrm{SO}(3)$. We denote by $S_{\rho}^{2}$ the sphere of radius $\rho$. As usual, $G_{\eta}$ denotes the isotropy subgroup of $\eta$ with respect to the coadjoint action; in the case at hand, $G_{\eta}=\left\{\right.$ rotations about $\left.\eta_{l}\right\} \times\left\{\right.$ rotations about $\left.\eta_{r}\right\}$.

Proposition 3 (i) For any $\eta=\left(\eta_{l}, \eta_{r}\right) \in\left(\mathbb{R}^{3}\right)^{2}$ with $\eta_{l} \neq 0 \neq \eta_{r}$, the reduced phase space $\mathbf{J}^{-1}(\eta) / G_{\eta}$ can be identified with

$$
P_{\eta}=\mathcal{B} \times \mathbb{R}^{2} \times\left(S_{\left\|\eta_{l}\right\|}^{2} \times S_{\left\|\eta_{r}\right\|}^{2}\right)
$$

After this identification, the canonical projection $\pi_{\eta}: \mathbf{J}^{-1}(\eta) \rightarrow P_{\eta}$ is $\pi_{\eta}(b, c, U)=\left(b, c,\left(U_{l}^{T} \eta_{l}, U_{r}^{T} \eta_{r}\right)\right)$. The symplectic structure $\sigma_{\eta}$ on $P_{\eta}$ defined by the equality $i_{\eta}^{*} \sigma=\pi_{\eta}^{*} \sigma_{\eta}$ is

$$
\sigma_{\eta}(b, c, m)\left((v, w, m \times \omega),\left(v^{\prime}, w^{\prime}, m \times \omega^{\prime}\right)\right)=v \cdot w^{\prime}-v^{\prime} \cdot w-m \cdot \omega \times \omega^{\prime} .
$$

The reduced Hamiltonian $H_{\eta}: P_{\eta} \rightarrow \mathbb{R}$, defined by $H_{\eta} \circ \pi_{\eta}=H \circ i_{\eta}$, is

$$
H_{\eta}(b, c, m)=\frac{1}{2} c \cdot \mathbb{K}(b) c+\frac{1}{2} m \cdot \mathbb{J}(b) m+\mathcal{V}(b) .
$$

(ii) If $\eta=\left(\eta_{l}, 0\right)$ or $\eta=\left(0, \eta_{l}\right)$ with $\eta_{l} \neq 0$, then the reduced phase space can be identified with $\mathcal{B} \times \mathbb{R}^{2} \times S_{\left\|\eta_{l}\right\|}^{2}$ and one has $\pi_{\eta}\left(b, c, U_{l}\right)=\left(b, c, U_{l}^{T} \eta_{l}\right)$ and

$$
\begin{array}{r}
\sigma_{\eta}\left(b, c, m_{l}\right)\left(\left(v, w, m_{l} \times \omega_{l}\right),\left(v^{\prime}, w^{\prime}, m_{l} \times \omega_{l}^{\prime}\right)\right)=v \cdot w^{\prime}-v^{\prime} \cdot w-m_{l} \cdot \omega_{l} \times \omega_{l}^{\prime} \\
H_{\eta}\left(b, c, m_{l}\right)=\frac{1}{2} c \cdot \mathbb{K}(b) c+\frac{1}{2} m_{l} \cdot J_{1}(b) m_{l}+\mathcal{V}(b) .
\end{array}
$$

The reduced system generically has four degrees of freedom, but only three in the special case (ii), which arises in the case of the so-called "irrotational" Riemann ellipsoids. The case $\eta_{l}=\eta_{r}=0$ yields a reduced system with two degrees of freedom, the only equilibrium of which is the stationary sphere.

In view of the application of Nekhoroshev theory, we note that the reduced Hamiltonian $H_{\eta}$ is an analytic function. This is seen by observing that the self-gravitational potential can be written in the form

$$
\mathcal{V}(b)=-\frac{4 \pi g}{\sqrt{b_{1}^{2}-b_{3}^{2}}} F\left(\arccos \left(\frac{b_{3}}{b_{1}}\right) \mid \sqrt{\frac{b_{1}^{2}-b_{2}^{2}}{b_{1}^{2}-b_{3}^{2}}}\right)
$$

where $F(\varphi \mid k)$ is the incomplete elliptic integral of the first kind, which is analytic for $\varphi \in\left(0, \frac{\pi}{2}\right)$ and $k \in(0,1)$ (see [3], pg. 4-5, 299).

D. Equivalence and symmetry of reduced systems. As we now discuss, (i) certain classes of reduced systems are equivalent and (ii) the reduced systems have certain discrete symmetries. For the sake of conciseness, we consider here only the generic case, in which both $\eta_{l}$ and $\eta_{r}$ are nonzero. (The case in which one of the momenta vanishes is recovered with obvious modifications.) There are two distinct reasons for equivalence of different reduced systems:

E1. The reduced system $\left(P_{\eta}, H_{\eta}, \sigma_{\eta}\right)$ depends on $\eta=\left(\eta_{l}, \eta_{r}\right)$ only through the norms of $\eta_{l}$ and $\eta_{r}$. This equivalence reflects the invariance of the Dirichlet problem under spatial rotation. 
E2. Dedekind proved that if $F(t)$ is a solution of the Dirichlet problem, then $F(t)^{T}$ is also a solution [8]. (The proof follows immediately from the observations that $\mathbb{P}_{F^{T}} G^{T}=\left(\mathbb{P}_{F} G\right)^{T}$ for any matrix $G$ and that $U_{l} A U_{r}^{T}$ is a singular value decomposition of $F$ iff $U_{r} A U_{l}^{T}$ is a singular value decomposition of $F^{T}$.) Chandrasekhar calls 'adjoint' the two motions determined by $F(t)$ and $F(t)^{T}$. This invariance of the Dirichlet problem has a counterpart in the Hamiltonian formulation on the covering space $\mathcal{M}$, which is invariant under the symplectomorphism exchanging $U_{l}$ with $U_{r}$ and $m_{l}$ with $m_{r}$. This map takes solutions to solutions, but exchanges the level sets of $\mathbf{J}$. Correspondingly, the reduced systems for the values $\left(\eta_{l}, \eta_{r}\right)$ and $\left(\eta_{r}, \eta_{l}\right)$ of the momentum map are conjugated to one another by the diffeomorphism $\left(b, c,\left(m_{l}, m_{r}\right)\right) \mapsto\left(b, c,\left(m_{r}, m_{l}\right)\right)$.

Furthermore, each reduced system is invariant under two discrete actions:

i. The $\mathbb{Z}_{2}$-action $(b, c, m) \mapsto(b, c,-m)$, which reflects the invariance of the original system with respect to the combination of spatial reflection and time reversal.

ii. The $\mathbb{Z}_{4}$-action $(b, c, m) \mapsto\left(b, c, R_{i} m\right), i=0, \ldots, 3$, where $R_{0}, \ldots, R_{3}$ are given by (2.4) and $R_{i}\left(m_{l}, m_{r}\right)=\left(R_{i} m_{l}, R_{i} m_{r}\right)$. The points $\left(b, c, R_{i} m\right)$ are obtained from one another by a reflection about one and the same coordinate axis on the two spheres; see figure 11. Hence the $\mathbb{Z}_{4}$-orbit of the point $(b, c, m)$ consists of two distinct points if $m$ is parallel to a basis vector $\left(e_{j}, e_{j}\right), j=1,2,3$, and of four distinct points otherwise.

In the stability analysis, we need not distinguish equivalent reduced systems, nor distinct points within the $\mathbb{Z}_{4} \times \mathbb{Z}_{2}$ orbits, in each reduced system. A $\mathbb{Z}_{4} \times \mathbb{Z}_{2}$-orbit consists of eight, four, or two points, depending on the number of nonzero components of $m_{l}$ and $m_{r}$. We will say that all points in such an orbit are $\mathbb{Z}_{4} \times \mathbb{Z}_{2}$-equivalent and regard two points in the reduced space as adjoint if any two points in their $\mathbb{Z}_{4} \times \mathbb{Z}_{2}$-orbits are adjoint.

By a Riemann ellipsoid we will mean an equivalence class, under spatial rotations (E1) and transposition (E2), of motions of the Dirichlet problem corresponding to an equilibrium of the reduced system. Thus each Riemann ellipsoid can be identified with the $\mathbb{Z}_{4} \times \mathbb{Z}_{2}$-orbit of an equilibrium of some reduced system. . $^{2}$

Remark: The $\mathbb{Z}_{4}$-symmetry is not present in Dirichlet's system (2.2), but is clearly inherited from the passage to the fourfold covering manifold $\mathcal{M}$ of $T^{*} Q$. (The existence of this symmetry of Riemann's equation is mentioned by Chandrasekhar ([6], pg. 72), but its origin is not identified there.) Since all points in a $\mathbb{Z}_{4}$-orbit correspond to the same state of the fluid, the passage to the covering introduces some redundancy, but allows us to avoid singular reduction. In fact, Dirichlet's equations (2.2) determine a $\mathrm{SO}(3) \times \mathrm{SO}(3)$-invariant Hamiltonian system on $T^{*} Q$; however, the action is not free and the reduced phase space is the orbifold $\mathcal{B} \times \mathbb{R}^{2} \times\left(S^{2} \times S^{2}\right) / \mathbb{Z}^{4}$, rather than a smooth manifold. The singular set consists of all states in which $m_{l}$ and $m_{r}$ are both parallel to the same axis of the ellipsoid, and thus contains the Riemann ellipsoids of type S.

\section{$3 \quad$ Riemann Ellipsoids}

We now study the equilibria of the reduced systems of Proposition 3, and thus the Riemann ellipsoids. The results of this analysis are due to Riemann. As we mentioned in the Introduction, there are five classes of Riemann ellipsoids and the momenta $m_{l}$ and $m_{r}$ of the ellipsoids of each type are completely determined by their semiaxes. Correspondingly, in the set $\mathcal{B}$ there are "existence regions" of the Riemann ellipsoids of each type. In order to specify these regions, and the momenta

\footnotetext{
${ }^{2}$ Note that, as was previously stated, we exclude from our consideration all axisymmetric ellipsoids.
} 


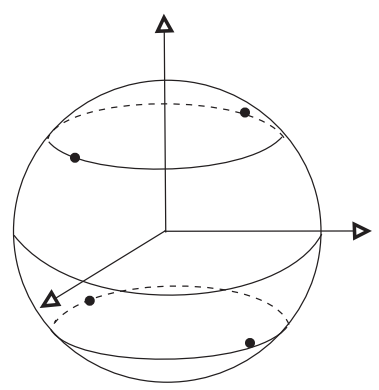

Figure 1: Projection of a $\mathbb{Z}_{4}$-orbit on one of the spheres.

of the corresponding Riemann ellipsoids, we introduce some notation. First we set

$$
C_{n}(x, y, z)=2 \pi g \int_{0}^{\infty}\left[\left(s+x^{2}\right)\left(s+y^{2}\right)\left(s+z^{2}\right)\right]^{-3 / 2} s^{n} d s, \quad n=0,1,2 .
$$

The functions

$$
G_{ \pm}^{S}(x, y, z)=\frac{(x \mp z)^{4}}{x z}\left[\left[x y^{2} z \pm\left(x^{2} y^{2}-x^{2} z^{2}+y^{2} z^{2}\right)\right] C_{1}(x, y, z)+\left(x z \pm y^{2}\right) C_{2}(x, y, z)\right]
$$

determine the momenta of the ellipsoids of type $\mathrm{S}$, while the functions

$$
\begin{aligned}
G(x, y, z) & =x^{2}\left(y^{2}-z^{2}\right) C_{1}(x, y, z)+\left(y^{2}-4 z^{2}\right)\left[z^{2} C_{1}(x, y, z)+C_{2}(x, y, z)\right] \\
D(x, y, z) & =x^{2}\left(y^{2}-z^{2}\right)+z^{2}\left(4 z^{2}-y^{2}\right) \\
G_{ \pm}^{R}(x, y, z) & =(y \mp z)^{4}\left(x^{2}-(y \pm 2 z)^{2}\right) \frac{x^{2}-z^{2}}{x^{2}-y^{2}} \frac{G(x, y, z)}{D(x, y, z)}
\end{aligned}
$$

determine the momenta of the ellipsoids of type I, II and III; we refer to the latter three classes of ellipsoids collectively as type $\mathrm{R}$ ellipsoids. 3 Using these functions we define the five sets

$$
\begin{aligned}
\mathcal{B}_{\mathrm{S}_{2}} & =\left\{b \in \mathcal{B}: G_{-}^{S}\left(b_{1}, b_{2}, b_{3}\right) \geq 0\right\} \\
\mathcal{B}_{\mathrm{S}_{3}} & =\left\{b \in \mathcal{B}: G_{+}^{S}\left(b_{1}, b_{3}, b_{2}\right) \geq 0\right\} \\
\mathcal{B}_{\mathrm{I}} & =\left\{b \in \mathcal{B}: b_{1} \leq 2 b_{2}-b_{3}\right\} \\
\mathcal{B}_{\mathrm{II}} & =\left\{b \in \mathcal{B}: b_{1} \geq 2 b_{2}+b_{3}, D\left(b_{1}, b_{3}, b_{2}\right)<0\right\} \\
\mathcal{B}_{\mathrm{III}} & =\left\{b \in \mathcal{B}: b_{1} \geq b_{2}+2 b_{3}, G\left(b_{1}, b_{2}, b_{3}\right)>0\right\}
\end{aligned}
$$

and five pairs of real-valued maps $\mu_{\alpha}^{ \pm}$, one for each type $\alpha$ of ellipsoids; these maps and their domains are described in Table 1 , where $\left\{e_{1}, e_{2}, e_{3}\right\}$ denotes the standard basis vector of $\mathbb{R}^{3}$ and

$$
N_{ \pm}^{\tau}(x, y, z)=\frac{1}{2}\left[\sqrt{G_{+}^{\tau}(x, y, z)} \pm \sqrt{G_{-}^{\tau}(x, y, z)}\right] \quad \tau=R, S .
$$

\footnotetext{
${ }^{3}$ In Riemann's article, the study of the existence of these ellipsoids precedes that of the ellipsoids of type S.
} 


\begin{tabular}{||c|c|c||}
\hline \hline Type $(\alpha)$ & Domain & $\mu_{\alpha}^{ \pm}(b)$ \\
\hline \hline $\mathrm{S}_{2}$ & $\mathcal{B}_{\mathrm{S}_{2}}$ & $N_{ \pm}^{S}\left(b_{1}, b_{2}, b_{3}\right) e_{2}$ \\
\hline $\mathrm{S}_{3}$ & $\mathcal{B}_{\mathrm{S}_{3}}$ & $N_{ \pm}^{S}\left(b_{1}, b_{3}, b_{2}\right) e_{3}$ \\
\hline $\mathrm{I}$ & $\mathcal{B}_{\mathrm{I}}$ & $N_{ \pm}^{R}\left(b_{1}, b_{3}, b_{2}\right) e_{1}+N_{ \pm}^{R}\left(b_{3}, b_{1}, b_{2}\right) e_{3}$ \\
\hline $\mathrm{II}$ & $\mathcal{B}_{\mathrm{II}}$ & $N_{ \pm}^{R}\left(b_{1}, b_{3}, b_{2}\right) e_{1}+N_{ \pm}^{R}\left(b_{3}, b_{1}, b_{2}\right) e_{3}$ \\
\hline $\mathrm{III}$ & $\mathcal{B}_{\mathrm{III}}$ & $N_{ \pm}^{R}\left(b_{1}, b_{2}, b_{3}\right) e_{1}+N_{\mp}^{R}\left(b_{2}, b_{1}, b_{3}\right) e_{2}$ \\
\hline \hline
\end{tabular}

Table 1: The momenta of the Riemann Ellipsoids

Proposition 4 (i) A point $(b, c, m) \in P_{\eta}$ is an equilibrium of the reduced system $\left(P_{\eta}, H_{\eta}, \sigma_{\eta}\right)$, $\eta_{l} \neq 0 \neq \eta_{r}$, iff it is $\mathbb{Z}_{4} \times \mathbb{Z}_{2}$-equivalent to either the point $\left(b, 0,\left(\mu_{\alpha}^{+}(b), \mu_{\alpha}^{-}(b)\right)\right)$ or the point $\left(b, 0,\left(\mu_{\alpha}^{-}(b), \mu_{\alpha}^{+}(b)\right)\right)$ for some $b \in \mathcal{B}_{\alpha}$ and some $\alpha=\mathrm{S}_{2}, \mathrm{~S}_{3}, \mathrm{I}, \mathrm{II}, \mathrm{III}$.

(ii) If one component of $\eta$ equals zero, then the equilibria of the six-dimensional reduced system are the points $\mathbb{Z}_{4} \times \mathbb{Z}_{2}$-equivalent to $\left(b, 0, \mu_{\alpha}^{+}(b)\right)$. This situation is met only at the equilibria of type $\mathrm{S}_{2}$ satisfying $G_{+}^{S}\left(b_{1}, b_{2}, b_{3}\right)=G_{-}^{S}\left(b_{1}, b_{2}, b_{3}\right)$ and those of type I satisfying $b_{1}^{2} b_{2}^{2}+b_{1}^{2} b_{3}^{2}+b_{2}^{2} b_{3}^{2}-3 b_{2}^{4}=0$; in both cases, $\mu_{\alpha}^{+}(b) \neq 0=\mu_{\alpha}^{-}(b)$.

The proof of this proposition can be reconstructed from Riemann's and Chandrasekhar's work. A sketch of the argument is given in Appendix A.

The existence regions (3.1) are drawn in figures 2 using the coordinates $(x, y)=\left(b_{2} / b_{1}, b_{3} / b_{1}\right)$, which have the advantage that the unbounded region $\mathcal{B}$ is mapped onto the bounded triangle $\{(x, y): 0<y<x<1\}$. The borders of the various regions are determined by the definitions (3.1). Note that, since the existence regions of ellipsoids of different types have nonempty intersections, there exist Riemann ellipsoids of up to four different types having equal semiaxes.

The Riemann ellipsoids belonging to a six-dimensional reduced space have either zero angular momentum or zero circulation and are called "irrotational" by Chandrasekhar. The existence of irrotational ellipsoids of type $\mathrm{S}_{2}$ was known to Riemann, while the existence of those of type I seems not to have been noticed before. Note that the curve of irrotational ellipsoids of type $\mathrm{S}_{2}$ divides the region $\mathcal{B}_{\mathrm{S}_{2}}$ into two parts, in one of which the two momenta are coparallel, while in the other they are counterparallel.

Remark: Since Chandrasekhar requires that the relative pressure be positive inside the ellipsoids, his region of existence of the ellipsoids of type II is smaller than ours, lying below the "zero-pressure curve" $D\left(b_{1}, b_{3}, b_{2}\right) C_{0}+6 b_{2}^{2} C_{1}+3 C_{2}=0$ shown in figure 2.c (see [6], chapter 7 , formula (195)).

\section{Stability and Ellipticity of the Riemann Ellipsoids}

A. Results. We now review the known results about the Lyapunov stability of the Riemann ellipsoids, regarded here as equilibria in the reduced phase space, and we describe the ellipticity analysis. From now on, we restrict ourselves to the generic case in which the reduced system has four degrees of freedom; the irrotational Riemann ellipsoids will be studied elsewhere.

\footnotetext{
${ }^{4}$ We say that two vectors $u$ and $v$ are coparallel (counterparallel) if $u \cdot v=\|u\|\|v\|(=-\|u\|\|v\|)$.
} 


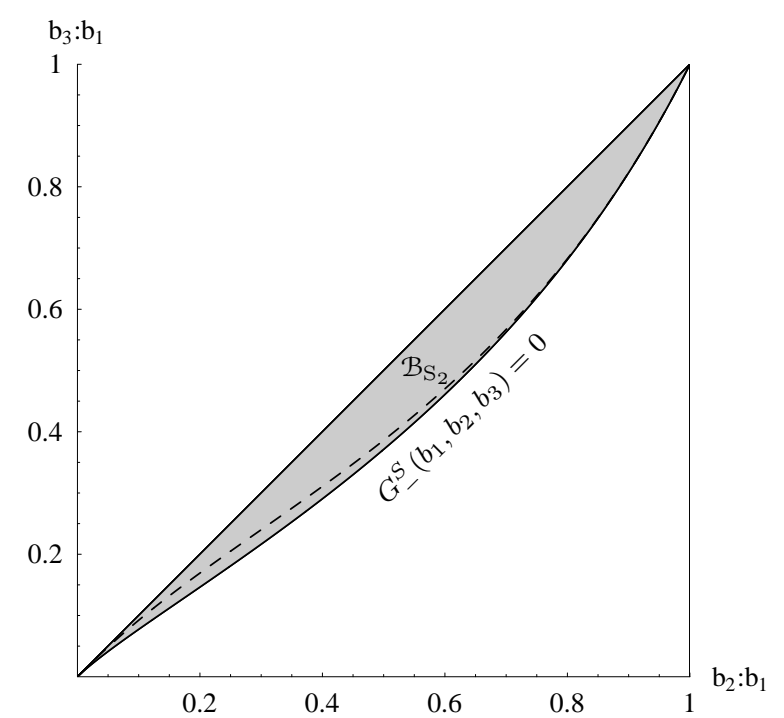

(a)

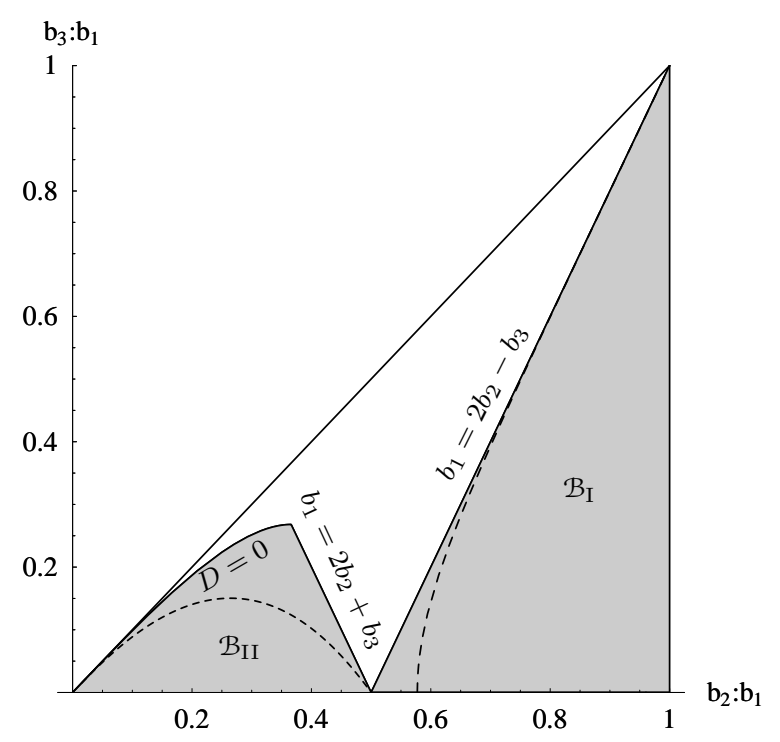

(c)

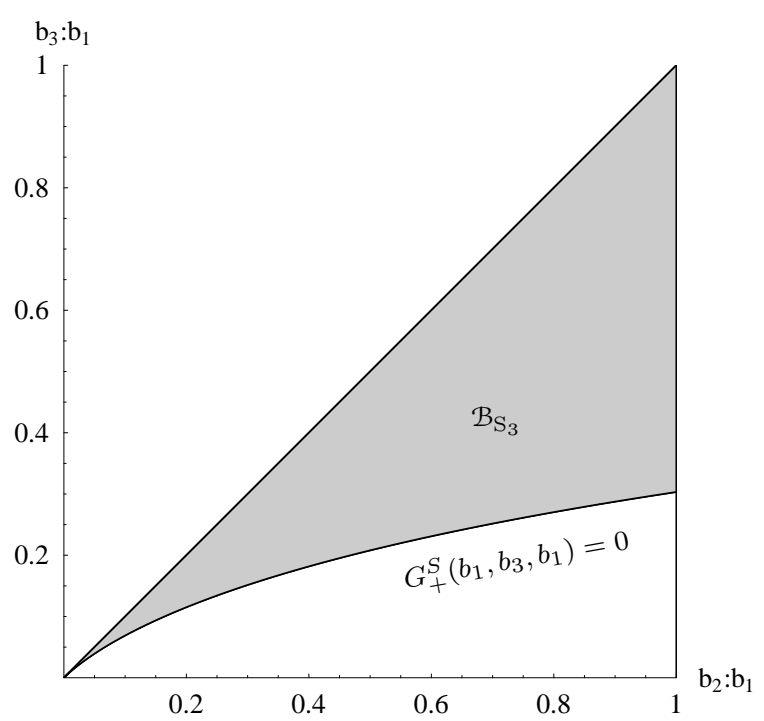

(b)

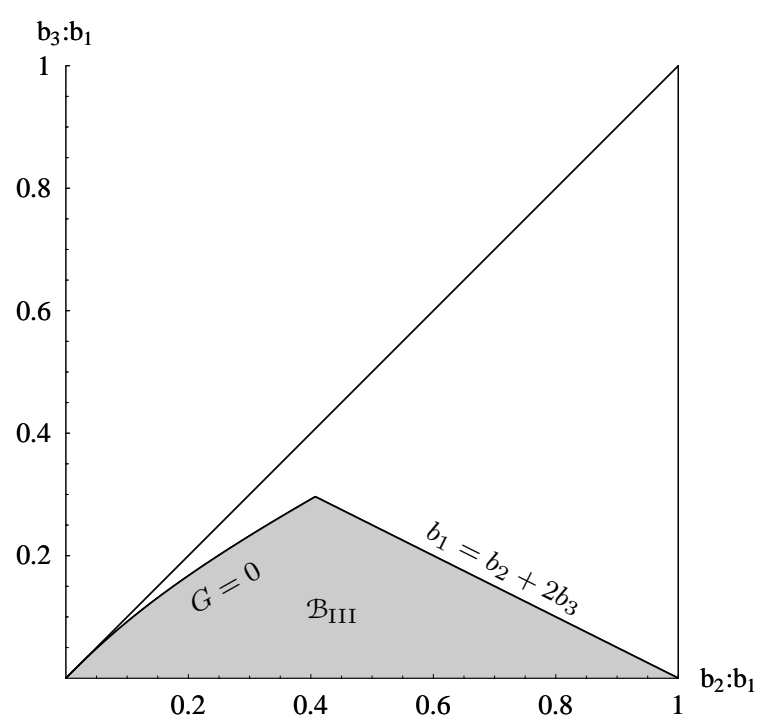

(d)

Figure 2: The existence regions of the five types of Riemann ellipsoids. The dashed curves in regions $\mathcal{B}_{\mathrm{S}_{2}}$ and $\mathcal{B}_{\text {I }}$ identify the irrotational ellipsoids and their adjoints (for which $\nu_{\alpha}=0$ ); the former separates the coparallel (lower) and the counterparallel (upper) $\mathrm{S}_{2}$-ellipsoids. The dashed curve in region $\mathcal{B}_{\text {II }}$ identifies the zero-pressure ellipsoids. 


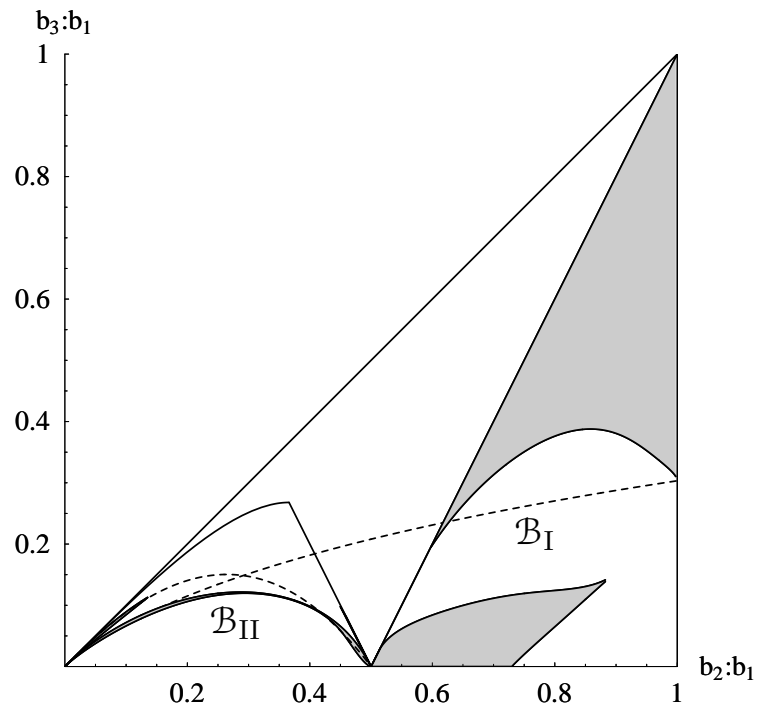

(a)

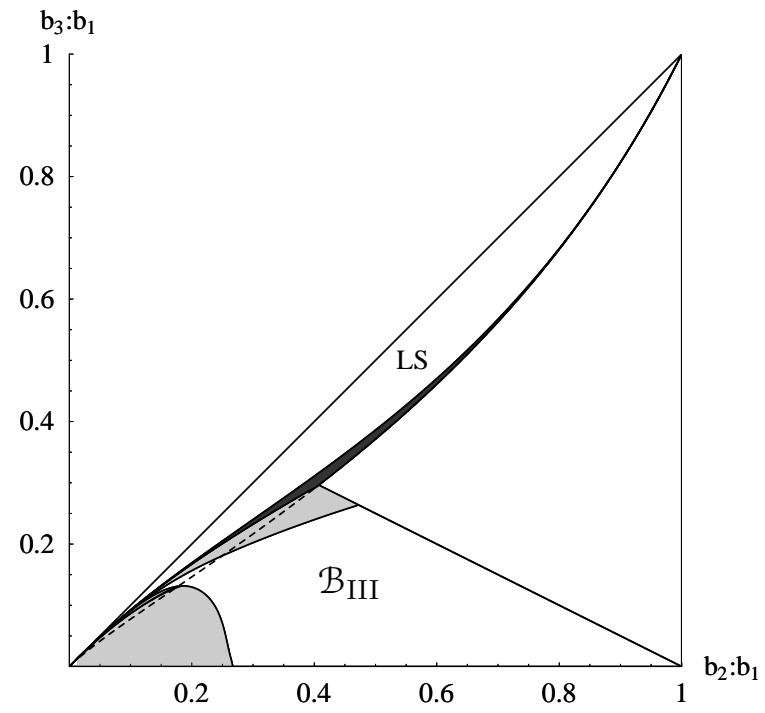

(b)

Figure 3: Regions of ellipticity of the Riemann ellipsoids of types I, II and III (light gray) and of the ellipsoids of type $\mathrm{S}_{2}$ (dark gray). The dashed curve contained in region $\mathcal{B}_{\text {II }}$ is the zero-pressure curve, while the one crossing regions $\mathcal{B}_{\mathrm{II}}$ and $\mathcal{B}_{\mathrm{I}}$ is the border of the existence region of the ellipsoids $\mathrm{S}_{3}$; the dashed curve in (b) is the border of the existence region of the $\mathrm{S}_{2}$. The Riemann ellipsoids of types I, II and III in the unshaded subregions of their existence regions are not elliptic and hence are Lyapunov unstable. The counterparallel $S_{2}$ ellipsoids, which lie in the unshaded subregion of $\mathcal{B}_{\mathrm{S}_{2}}$ marked LS, are Lyapunov stable; the coparallel $\mathrm{S}_{2}$ ellipsoids in $\mathcal{B}_{\mathrm{S}_{2}} \cap \operatorname{Int}\left(\mathcal{B}_{\mathrm{III}}\right)$ are Lyapunov unstable (in figure (b), this part of region $\mathcal{B}_{\mathrm{S}_{2}}$ is 'hidden' below $\mathcal{B}_{\text {III }}$ ).

Proposition 5 (i) All $\mathrm{S}_{3}$-ellipsoids and all counterparallel $\mathrm{S}_{2}$-ellipsoids are Lyapunov stable.

(ii) The coparallel $\mathrm{S}_{2}$-ellipsoids for $b \in \mathcal{B}_{\mathrm{S}_{2}} \cap \operatorname{Int}\left(\mathcal{B}_{\mathrm{III}}\right)$ (i.e., $G\left(b_{1}, b_{2}, b_{3}\right)>0$ ) are not elliptic and hence are Lyapunov unstable.

The first statement is due to Riemann [20], who proved it using arguments essentially equivalent to using the reduced Hamiltonian as a Lyapunov function. Riemann also showed that the reduced Hamiltonian has a saddle point, and thus cannot be used as a Lyapunov function, at any other ellipsoid. Riemann interpreted this fact as an indication that these ellipsoids are unstable ("labil"). However, as best we know this fact is unproven, except in the case of the $\mathrm{S}_{2}$ ellipsoids considered in statement (ii), the instability of which follows from Chandrasekhar's work, since he showed that the linearization of the equations of motion has an eigenvalue with nonzero real part at these points; see section 4.B.

Remark: Given the structure of the problem, the Lyapunov stability of the equilibria of the reduced system corresponds to orbital stability of the solutions of the Dirichlet problem (see e.g. [19], [13], [18]).

The ellipticity of the Riemann ellipsoids has been studied by Chandrasekhar; most of his results are based on numeric calculations. We have repeated the numerical analysis of the ellipticity, finding the same results as Chandrasekhar for the $\mathrm{S}_{2}$ ellipsoids and essentially the same results for the ellipsoids of type I, but significantly different results for the ellipsoids of types II and III. The conclusions of our investigation are the following: 
Numerical Conclusions 1: (i) The coparallel $\mathrm{S}_{2}$-ellipsoids for $b$ in the complement of $\mathcal{B}_{\mathrm{S}_{2}} \cap$ $\operatorname{Int}\left(\mathcal{B}_{\text {III }}\right)$, i.e. $b$ satisfying $G\left(b_{1}, b_{2}, b_{3}\right) \leq 0$, are elliptic.

(ii) There are nonempty subregions of $\mathcal{B}_{\mathrm{I}}, \mathcal{B}_{\mathrm{II}}$ and $\mathcal{B}_{\mathrm{III}}$ consisting of elliptic ellipsoids; these regions are the shaded regions in figures 3 and 6 . The ellipsoids of types I, II and III in the complement of these subregions are not elliptic and hence are Lyapunov unstable.

This analysis has been carried out by analytically constructing the linearization of the reduced Hamiltonian vector field at the reduced equilibria and by numerically evaluating this matrix at some tens of thousands of ellipsoids of each type. Details about this analysis are given in the next subsection. (As explained there, Riemann sketches an argument that would imply statement (i).) Here we comment on these results.

- The elliptic $\mathrm{S}_{2}$ ellipsoids of unknown Lyapunov stability lie between the "irrotational" curve and the border of region $\mathcal{B}_{\mathrm{III}}$. This is the dark shaded region in figure 3.b.

- Our computations confirm the existence of two ellipticity regions for the ellipsoids of type I, as shown in figure 3 of [5], but disclose a finer structure of the lower region than that found by Chandrasekhar: the upper border of this region appears to transversally intersect the border $b_{1}=2 b_{2}-b_{3}$ of the existence region at about $b_{2} / b_{1}=.515$, but there is a very narrow crescent of nonelliptic ellipsoids along this line, approximately in the range $.5<b_{2} / b_{1}<.503$, which is shown in figure 1 .a. We could not determine with certainty if this crescent actually touches the line $b_{1}=2 b_{2}-b_{3}$.

- The region of elliptic ellipsoids of type II consists of three narrow fringes, shown enlarged in figure $1 . \mathrm{b}, \mathrm{c}, \mathrm{d}$. None of these regions were detected by Chandrasekhar, who stated that no ellipsoid of type II is elliptic ([5], pg. 169). Note that two of these fringes belong (for the most part) to the region of positive relative pressure considered by Chandrasekhar.

- The region of ellipticity of the ellipsoids of type III has a three-lobed structure, the three lobes being separated by two very thin gaps; see figure 1. .e, f. Chandrasekhar detected only the upper of these three lobes. To within the precision of our numerical computations, the two gaps close at approximately $b_{2} / b_{1}=.01$, but it is possible that, in fact, they persist to the origin.

In an attempt to understand the discrepancies between our results and Chandrasekhar's, we tested our calculations using his linearization of the equations of motion, finding complete agreement. It is therefore possible that Chandrasekhar's conclusions were simply based on too few sample cases to obtain a detailed global picture of the structure of the regions of ellipticity. A more detailed comparison with Chandrasekhar's result is given in Appendix B.

We conclude this analysis by observing that the sources of the richness and intricacy of the structure of the ellipticity regions resulting from these numerical computations are not yet understood. Of course, we cannot be sure that our numerical computations, even though accurate, ultimately detected the finest details of this structure, particularly in view of the nontrivial structure of the ellipticity regions for ellipsoids approaching a degenerate (flat or rodlike) configuration. Many features remain to be understood. For instance, figures 3.a and 4. suggest that there are (nontrivial) relations between the ellipticity regions of the ellipsoids I (resp. III) and the boundary of the existence region of the ellipsoids $\mathrm{S}_{2}$ (resp. $\mathrm{S}_{3}$ ), which could be the object of a bifurcation analysis. Additional analytical insight into the problem is certainly needed.

Remarks: (i) In his articles, Chandrasekhar refers to ellipticity as "stability", while Riemann designated by this name what is now called Lyapunov stability. Because of this, and the previously mentioned fact that Riemann regarded as unstable the ellipsoids which are saddle points of the Lyapunov function, Riemann and Chandrasekhar found different "stability" and "instability" 

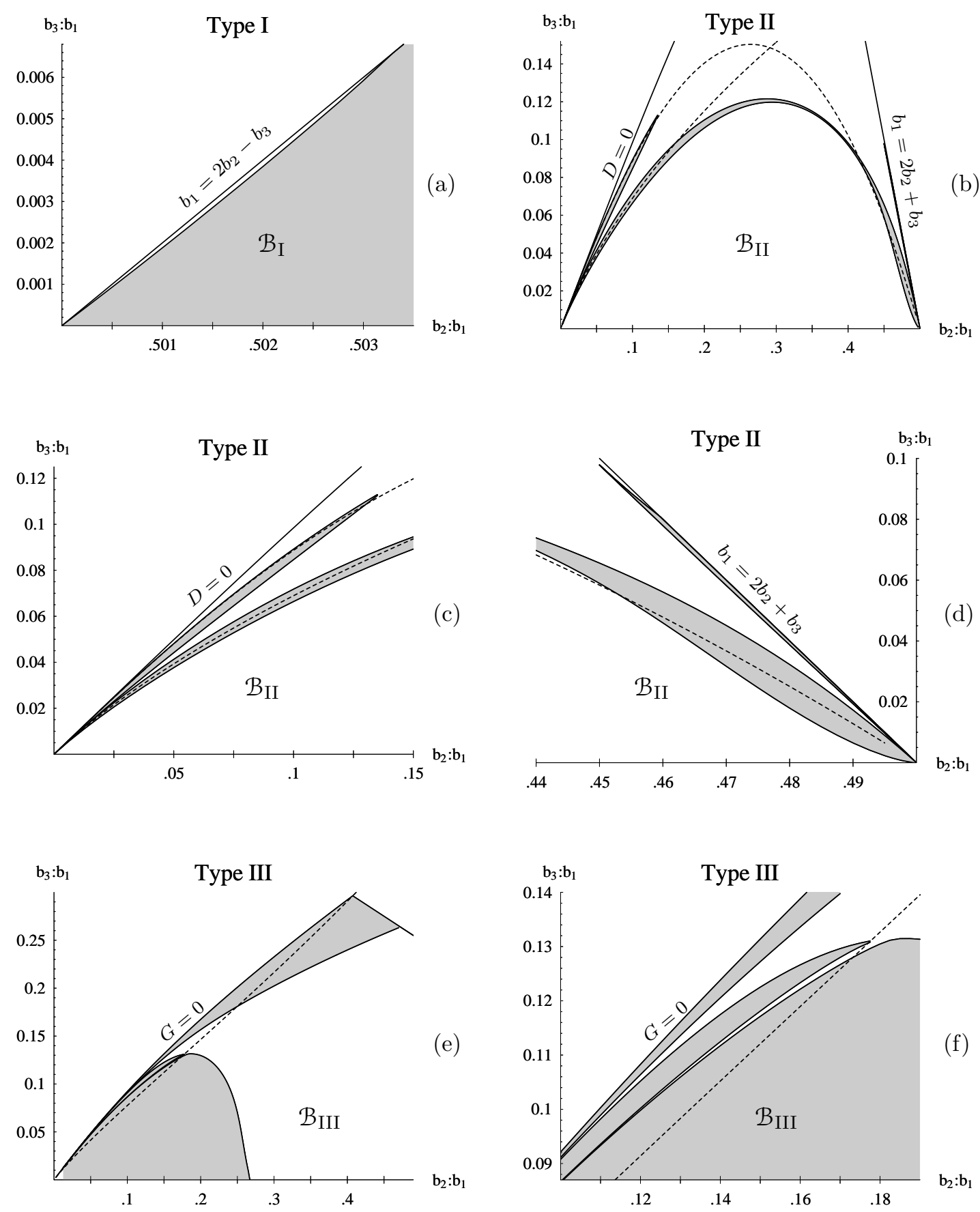

Figure 4: Details of the regions of ellipticity (shaded) of the ellipsoids of types I, II and III. All curves are as in figure 3 . 
regions. Chandrasekhar interpreted this difference as being due to some errors in Riemann's work (see page 875 in [5] and pages 185-7 in [6]). Riemann's results are fully confirmed by our numerical computations (with the exception of the characterization of the saddle points as unstable, of course) and are, in fact, compatible with Chandrasekhar's results once the distinction between Lyapunov stability and ellipticity is taken into account.

(ii) In the quoted references, Chandrasekhar refers to [12] for an explanation of "the origin of his [Riemann's] errors". In that article, to the best of our understanding, the source of these supposed errors is ascribed to the fact that Riemann treated the Dirichlet problem as a Lagrangian system (which is correct, as we have noted in Section 2), while according to [12] the system is Lagrangian only in the case of the ellipsoids of type S; remark (vi) in page 185 of [6] seems to indicate that Chandrasekhar shared this opinion.

B. Numerical Computations. We now briefly describe the numerical procedures used in the verification of Numerical Conclusions 1 and in the Nekhoroshev analysis. We also provide the key components of the proof of statement (ii) of Proposition 5. We use canonical coordinates obtained by introducing a pair of local "Poincaré coordinates" $(q, p)$ on each sphere in the reduced phase space; these coordinates coincide with the Poincaré elements used in the Kepler problem. The use of coordinates is not strictly necessary for the ellipticity analysis, but is very useful for the construction of the normal forms used in the analysis of the Nekhoroshev stability.

Lemma 1 For any $\rho>0$, the map

$$
(q, p) \mapsto \tilde{m}_{\rho}(q, p):=\left(p \sqrt{\rho-\frac{q^{2}+p^{2}}{4}},-q \sqrt{\rho-\frac{q^{2}+p^{2}}{4}}, \rho-\frac{q^{2}+p^{2}}{2}\right)
$$

is a diffeomorphism from the disk $\left\{(q, p) \in \mathbb{R}^{2}: q^{2}+p^{2}<2 \rho\right\}$ onto the half-sphere $\left\{m \in \mathbb{R}^{3}\right.$ : $\left.\|m\|=\rho, m_{3}>0\right\}$. It is symplectic in the sense that $\left(\tilde{m}_{\rho}\right)_{*}(d p \wedge d q)\left(m \times \omega, m \times \omega^{\prime}\right)=-m \cdot \omega \times \omega^{\prime}$ for all $m$ in the half sphere and all $\omega, \omega^{\prime} \in \mathbb{R}^{3}$.

Proof. The map (4.1) is smooth and onto; its inverse $m \mapsto \sqrt{2 /\left(\rho+m_{3}\right)}\left(-m_{2}, m_{1}\right)$ is also smooth. If $v=\left(v_{1}, v_{2}, v_{3}\right)$ is tangent to the sphere at the point $m=\tilde{m}_{\rho}(q, p)$, then $v=v_{q} \frac{\partial \tilde{m}}{\partial q}+v_{p} \frac{\partial \tilde{m}}{\partial p}$ for some constants $v_{q}$ and $v_{p}$; specifically, if we set $k_{1}=2 \rho-q^{2}-p^{2}$ and $k_{2}=k_{1}+2 \rho$, then $k_{1} \sqrt{k_{2}} v_{q}=q p v_{1}-\left(k_{2}-p^{2}\right) v_{2}$ and $k_{1} \sqrt{k_{2}} v_{p}=\left(k_{2}-q^{2}\right) v_{1}-q p v_{2}$. Thus $\left(\tilde{m}_{\rho}\right)_{*}(d p \wedge d q)\left(m \times \omega, m \times \omega^{\prime}\right)=$ $(m \times \omega)_{p}\left(m \times \omega^{\prime}\right)_{q}-(m \times \omega)_{q}\left(m \times \omega^{\prime}\right)_{p}=-m \cdot\left(\omega \times \omega^{\prime}\right)$ for any $\omega, \omega^{\prime} \in \mathbb{R}^{3}$.

When studying a given equilibrium $\left(b^{*}, 0, M^{*}\right)$, with $M^{*}=\left(m_{l}^{*}, m_{r}^{*}\right)$, we shall translate the origin of the coordinates $b$ to the equilibrium value $b^{*}$; for simplicity, we will retain the notation $b$ for the translated coordinates. Moreover, we shall use 'rotated' Poincaré coordinates $\left(q_{1}, p_{1}\right)$ and $\left(q_{2}, p_{2}\right)$ centered at $m_{l}^{*}$ and $m_{r}^{*}$ on the spheres; specifically, for given $m^{*} \in \mathbb{R}^{3}$ we define

$$
(q, p) \mapsto \tilde{m}\left(q, p ; m^{*}\right):=R_{m^{*}} \tilde{m}_{\left\|m^{*}\right\|}(q, p)
$$

where $R_{m^{*}}$ is an orthogonal matrix such that $R_{m^{*}} e_{3}=\frac{m^{*}}{\left\|m^{*}\right\|}$. Since rotations are symplectic, the coordinates $\tilde{m}\left(\cdot ; m^{*}\right)$ are symplectic. These coordinates are not uniquely defined: rightmultiplication of $R_{m^{*}}$ by a rotation about $e_{3}$ produces a new system of rotated Poincaré coordinates 
centered at $m^{*}$. To compress the notation, we write $(q, p)$ for $\left(q_{1}, q_{2}, p_{1}, p_{2}\right)$ and

$$
M\left(q, p ; M^{*}\right)=\left(\tilde{m}\left(q_{1}, p_{1} ; m_{l}^{*}\right), \tilde{m}\left(q_{2}, p_{2} ; m_{r}^{*}\right)\right) .
$$

Thus, the reduced Hamiltonian (2.8) takes the form

$$
\mathcal{H}\left(b, c, q, p ; b^{*}, M^{*}\right)=\frac{1}{2} c \cdot \mathbb{K}\left(b^{*}+b\right) c+\frac{1}{2} M\left(q, p ; M^{*}\right) \cdot \mathbb{J}\left(b^{*}+b\right) M\left(q, p ; M^{*}\right)+\mathcal{V}\left(b^{*}+b\right) .
$$

We now study the block structure of the Hessian matrix $\mathcal{H}^{\prime \prime}\left(0 ; b^{*}, M^{*}\right)$. To obtain a Hessian with as few nontrivial blocks as possible, we construct the coordinates using matrices such that $R_{m_{l}^{*}} e_{1}=R_{m_{r}^{*}} e_{1}$ is parallel to a principal axis orthogonal to $\operatorname{span}\left[m_{l}, m_{r}\right]$. Using this choice of coordinates and denoting by $\mathcal{H}_{b b}^{\prime \prime}$ the two-by-two block $\frac{\partial^{2} \mathcal{H}}{\partial b_{i} \partial b_{j}}$, etc, we have

Lemma 2 For all equilibria, the blocks $\mathcal{H}_{c b}^{\prime \prime}, \mathcal{H}_{c q}^{\prime \prime}, \mathcal{H}_{c p}^{\prime \prime}, \mathcal{H}_{b p}^{\prime \prime}$, and $\mathcal{H}_{q p}^{\prime \prime}$ equal zero and $\mathcal{H}_{c c}^{\prime \prime}=\mathbb{K}$. Moreover, $\mathcal{H}_{b q}^{\prime \prime}=0$ for the equilibria of type $S$.

Proof. The equalities $\mathcal{H}_{c b}^{\prime \prime}=\mathcal{H}_{c q}^{\prime \prime}=\mathcal{H}_{c p}^{\prime \prime}=0$ and $\mathcal{H}_{c c}^{\prime \prime}=\mathbb{K}$ are obvious. Note that the 'standard' Poincaré parameterization (4.1) of the sphere satisfies

$$
\frac{\partial \tilde{m}}{\partial q}(0,0 ; \rho)=-\sqrt{\rho} e_{2}, \quad \frac{\partial \tilde{m}}{\partial p}(0,0 ; \rho)=\sqrt{\rho} e_{1}, \quad \frac{\partial^{2} \tilde{m}}{\partial q \partial p}(0,0 ; \rho)=0 .
$$

Using the latter equality and denoting by $\frac{\partial \tilde{M}}{\partial q_{j}}$ (resp. $\frac{\partial \mathbb{J}}{\partial q_{j}}$ ) the vector (resp. matrix) with entries given by the derivatives with respect to $q_{j}$ of the entries of $\tilde{M}$ (resp. $\mathbb{J}$ ), etc., we have

$$
\begin{aligned}
\frac{\partial^{2} \mathcal{H}}{\partial b_{i} \partial p_{j}}\left(0 ; b^{*}, M^{*}\right) & =R \frac{\partial \tilde{M}}{\partial p_{j}}(0,0) \cdot \frac{\partial \mathbb{J}}{\partial b_{i}}\left(b^{*}\right) M^{*} \\
\frac{\partial^{2} \mathcal{H}}{\partial q_{i} \partial p_{j}}\left(0 ; b^{*}, M^{*}\right) & =R \frac{\partial \tilde{M}}{\partial q_{i}}(0,0) \cdot \mathbb{J}\left(b^{*}\right) R \frac{\partial \tilde{M}}{\partial p_{j}}(0,0) \\
\frac{\partial^{2} \mathcal{H}}{\partial b_{i} \partial q_{j}}\left(0 ; b^{*}, M^{*}\right) & =R \frac{\partial \tilde{M}}{\partial q_{j}}(0,0) \cdot \frac{\partial \mathbb{J}}{\partial b_{i}}\left(b^{*}\right) M^{*}
\end{aligned}
$$

where $R$ is the six-by-six block diagonal matrix with diagonal blocks $R_{m_{l}^{*}}$ and $R_{m_{r}^{*}}$.

Due to the block structure of $\mathbb{J}$, the two 'components' of $\frac{\partial \mathbb{J}}{\partial b_{i}}\left(b^{*}\right) M^{*}$ lie in $\operatorname{span}\left[m_{l}, m_{r}\right]$. (Here we refer to $m_{l}$ and $m_{r}$ as the 'components' of $\left(m_{l}, m_{r}\right)$.) On the other hand, (4.2) and the condition that $R_{m_{l}^{*}} e_{1}=R_{m_{r}^{*}} e_{1}$ be orthogonal to $\operatorname{span}\left[m_{l}, m_{r}\right]$ imply that the components of $R \frac{\partial \tilde{M}}{\partial p_{j}}(0,0)$ are orthogonal to $\operatorname{span}\left[m_{l}, m_{r}\right]$ for $j=1,2$; hence (4.3) implies that $\frac{\partial^{2} \mathcal{H}}{\partial b_{i} \partial p_{j}}\left(0 ; b^{*}, M^{*}\right)=0$. To see that $\frac{\partial^{2} \mathcal{H}}{\partial q_{i} \partial p_{j}}\left(0 ; b^{*}, M^{*}\right)$ equals zero, note that $R_{m_{l}^{*}} e_{1}=R_{m_{r}^{*}} e_{1}$ is, by construction, an eigenvector of $J_{1}\left(b^{*}\right)$ and $J_{2}\left(b^{*}\right)$; hence (4.2) implies that the components of $R^{T} \mathbb{J}\left(b^{*}\right) R \frac{\partial \tilde{M}}{\partial p_{j}}(0,0)$ are orthogonal to the components of $\frac{\partial \tilde{M}}{\partial q_{j}}(0,0)$.

In the case of the $\mathrm{S}$ ellipsoids, the two components of $M^{*}$ are both parallel to the same coordinate axis. Due to the block structure of $\mathbb{J}$, the components of $\frac{\partial \mathbb{J}}{\partial b_{i}}\left(b^{*}\right) M^{*}$ are parallel to the components

\footnotetext{
${ }^{5}$ In what follows, to keep the notation simple, we will write all vectors as row vectors, even though they must be regarded as column vectors in all matrix formulas.
} 
of $M^{*}$; thus, both components of $R^{T} \frac{\partial J}{\partial b_{i}} M^{*}$ are parallel to $e_{3}$. By (4.2) and (4.3), this implies $\mathcal{H}_{b q}^{\prime \prime}=0$.

Let us now consider the ellipsoids of type $\mathrm{S}_{2}$. With a suitable choice of the coordinates, the Hessian has the diagonal block structure

$$
\mathcal{H}^{\prime \prime}\left(0 ; b^{*}, M^{*}\right)=\operatorname{diag}\left[\mathcal{H}_{b b}^{\prime \prime}, \mathbb{K}, \mathcal{H}_{q q}^{\prime \prime}, \mathcal{H}_{p p}^{\prime \prime}\right] .
$$

We limit ourselves to stating without proof the following facts, which can be proven using elementary, though sometimes laborious, computations:

- The blocks $\mathbb{K}$ and $\mathcal{H}_{q q}^{\prime \prime}$ are positive definite for all the $\mathrm{S}_{2}$-ellipsoids.

- The block $\mathcal{H}_{p p}^{\prime \prime}$ is positive definite for all the counterparallel $\mathrm{S}_{2}$-ellipsoids and is indefinite for all the coparallel $\mathrm{S}_{2}$-ellipsoid.

The block $\mathcal{H}_{b b}^{\prime \prime}$ has a relatively simple expression, but the analytical study of its definiteness is quite laborious. We verified numerically that it is positive definite at all ellipsoids of type $\mathrm{S}_{2}$. (Chandrasekhar's study of this block in [4] is also numerical.) The linearization at the equilibrium of the Hamiltonian vector field of $\mathcal{H}$ is $X\left(b^{*}, M^{*}\right)(b, c, q, p)$, where the matrix $X\left(b^{*}, M^{*}\right)$ is the product of the symplectic matrix and $\mathcal{H}^{\prime \prime}\left(0 ; b^{*}, M^{*}\right)$. Thus, at a type $\mathrm{S}_{2}$ ellipsoid, the matrix $X\left(b^{*}, M^{*}\right)$ is block diagonal, with the two $4 \times 4$ blocks

$$
X_{b c}=\left(\begin{array}{cc}
0 & \mathbb{K} \\
-\mathcal{H}_{b b}^{\prime \prime} & 0
\end{array}\right) \quad \text { and } \quad X_{q p}=\left(\begin{array}{cc}
0 & \mathcal{H}_{p p}^{\prime \prime} \\
-\mathcal{H}_{q q}^{\prime \prime} & 0
\end{array}\right)
$$

Since the eigenvalues of each of these two matrices are purely imaginary if its two nonzero blocks are positive semi-definite, we arrive to statement (i) of Numerical Conclusions 1. This conclusion is not rigorous because it is based on the numerical verification of the definiteness of $\mathcal{H}_{b b}^{\prime \prime}$. Statement (ii) of Proposition 5 follows from the definiteness of $\mathcal{H}_{q q}^{\prime \prime}$ and the indefiniteness of $\mathcal{H}_{p p}^{\prime \prime}$.

Remark: In [20], Riemann suggests an analytic argument that would show that for all ellipsoids of type $\mathrm{S}$ satisfying $m_{l} \neq m_{r}$, i.e. $G_{-}^{S}\left(b_{1}, b_{2}, b_{3}\right)>0$, the function $b \mapsto \mathcal{H}\left(b, 0,0,0 ; b^{*}, M^{*}\right)$ has a minimum at $b=0$. Since the block $\mathcal{H}_{b b}^{\prime \prime}$ is positive semi-definite at such a minimum, this result could be used to construct an analytic proof of statement (i) of Numerical Conclusions 1. However, as Riemann explicitly states, he does not actually carry out the minimality argument in [20].

We now consider the ellipsoids of types I, II and III. The Hessian has the form

$$
\mathcal{H}^{\prime \prime}\left(0 ; b^{*}, M^{*}\right)=\left(\begin{array}{cccc}
\mathcal{H}_{b b}^{\prime \prime} & 0 & \mathcal{H}_{b q}^{\prime \prime} & 0 \\
0 & \mathbb{K} & 0 & 0 \\
\mathcal{H}_{q b}^{\prime \prime} & 0 & \mathcal{H}_{q q}^{\prime \prime} & 0 \\
0 & 0 & 0 & \mathcal{H}_{p p}^{\prime \prime}
\end{array}\right)
$$

The eigenvalue problem of this matrix factorizes into the eigenvalue problems for the three matrices

$$
\mathbb{K}, \quad \mathcal{H}_{p p}^{\prime \prime}, \quad\left(\begin{array}{ll}
\mathcal{H}_{b b}^{\prime \prime} & \mathcal{H}_{b q}^{\prime \prime} \\
\mathcal{H}_{q b}^{\prime \prime} & \mathcal{H}_{q q}^{\prime \prime}
\end{array}\right) .
$$

One can prove that the two-by-two matrix $\mathcal{H}_{p p}^{\prime \prime}$ is indefinite for any ellipsoid of type I, II or III. (This implies that these ellipsoids are saddle points of the reduced Hamiltonian, as stated by Riemann.) The eigenvalue problem for the linearization of the Hamiltonian vector field determined by this Hessian does not factorize. Therefore the analysis of the ellipticity is particularly difficult. We know of no rigorous ellipticity results for these ellipsoids. 


\section{Nekhoroshev-stability of the Riemann-ellipsoids: Generalities}

We base our study of the Nekhoroshev-stability of the Riemann ellipsoids on some recent results in Nekhoroshev theory, which we describe in this section with reference to the problem at hand; complete proofs can be found in [2] (see also [10], [17], [11]). As is common in Hamiltonian perturbation theory, the procedure consists of the construction of the Birkhoff normal form of order four for the reduced Hamiltonian and testing of the normal form for certain properties that we now specify.

A. Birkhoff Normal Form. Consider an elliptic equilibrium $\left(b^{*}, M^{*}\right)$ with $M^{*}=\left(m_{l}^{*}, m_{r}^{*}\right)$, $m_{l}^{*} \neq 0 \neq m_{r}^{*}$. Use the canonical coordinates $\xi=(b, c, q, p)$ defined as in Section 4.B. Let $\mathcal{H}_{j}\left(\xi ; b^{*}, M^{*}\right)$ denote the $j$-th term in the Taylor series at $\xi=0$ for the reduced Hamiltonian $\mathcal{H}$, so that $\mathcal{H}_{j}$ is a homogeneous polynomial of order $j$ in $\xi$ and

$$
\mathcal{H}\left(\xi ; b^{*}, M^{*}\right)=\mathcal{H}_{2}\left(\xi ; b^{*}, M^{*}\right)+\mathcal{H}_{3}\left(\xi ; b^{*}, M^{*}\right)+\mathcal{H}_{4}\left(\xi ; b^{*}, M^{*}\right)+\ldots
$$

Let $X\left(b^{*}, M^{*}\right) \xi$ be the Hamiltonian vector field of $\mathcal{H}_{2}$. Since the equilibrium is elliptic, the matrix $X\left(b^{*}, M^{*}\right)$ has purely imaginary eigenvalues

$$
\pm i \omega_{j}\left(b^{*}, M^{*}\right), \quad j=1, \ldots, 4,
$$

with the convention $\omega_{j} \geq 0$.

The first step in the construction of the Birkhoff normal form for the Hamiltonian $\mathcal{H}$ is the "symplectic diagonalization" of the quadratic term $\mathcal{H}_{2}$. It is known that if the eigenvalues of $X\left(b^{*}, M^{*}\right)$ are all distinct, then there is a linear canonical change of coordinates

$$
\xi \mapsto \Xi=(B, C, Q, P)=T\left(b^{*}, M^{*}\right)^{-1} \xi
$$

such that $\widehat{\mathcal{H}}_{2}\left(\Xi ; b^{*}, M^{*}\right):=\mathcal{H}_{2}\left(T\left(b^{*}, M^{*}\right) \Xi ; b^{*}, M^{*}\right)$ has the form

$$
\widehat{\mathcal{H}}_{2}=s_{1} \omega_{1} \frac{B_{1}^{2}+C_{1}^{2}}{2}+s_{2} \omega_{1} \frac{B_{2}^{2}+C_{2}^{2}}{2}+s_{3} \omega_{3} \frac{Q_{1}^{2}+P_{1}^{2}}{2}+s_{4} \omega_{4} \frac{Q_{2}^{2}+P_{2}^{2}}{2},
$$

where $s_{j}= \pm 1$. The matrix $T$ and the numbers $s_{j}$ are constructed as follows: For $j=1, . ., 4$, let $x_{j}^{ \pm}=x_{j}^{\prime} \pm i x_{j}^{\prime \prime} \in \mathbb{C}^{8}$, with $x_{j}^{\prime}, x_{j}^{\prime \prime} \in \mathbb{R}^{8}$, denote any eigenvectors of the matrix $X\left(b^{*}, M^{*}\right)$ associated to the eigenvalues $\pm i \omega_{j}$. One can show that

$$
\Gamma_{j}\left(b^{*}, M^{*}\right):=x_{j}^{\prime} \cdot J_{8} x_{j}^{\prime \prime} \neq 0, \quad j=1, . ., 4,
$$

where $J_{8}=\operatorname{diag}\left[J_{4}, J_{4}\right]$; here $J_{4}$ denotes the standard four-by-four symplectic matrix $\left(\begin{array}{cc}\mathbf{0} & \mathbf{1} \\ -\mathbf{1} & \mathbf{0}\end{array}\right)$. We set $\left(u_{j}, v_{j}\right)=\left(x_{j}^{\prime}, x_{j}^{\prime \prime}\right)$ if $\Gamma_{j}>0$ and $\left(u_{j}, v_{j}\right)=\left(x_{j}^{\prime \prime}, x_{j}^{\prime}\right)$ if $\Gamma_{j}<0$, and define

$$
T=\left(\frac{u_{1}}{\gamma_{1}}, \frac{u_{2}}{\gamma_{2}}, \frac{v_{1}}{\gamma_{1}}, \frac{v_{2}}{\gamma_{2}}, \frac{u_{3}}{\gamma_{3}}, \frac{u_{4}}{\gamma_{4}}, \frac{v_{3}}{\gamma_{3}}, \frac{v_{4}}{\gamma_{4}}\right), \quad s_{j}=\operatorname{Sign}\left(\Gamma_{j}\right)
$$

where $\gamma_{j}=\sqrt{\left|\Gamma^{j}\right|}$; the notation means that the eight vectors are the rows of the matrix. Note that the condition that all the eigenvalues of $X$ be distinct (and hence nonzero) can be regarded as the condition that the "frequency vector"

$$
\Omega=\left(s_{1} \omega_{1}, \ldots, s_{4} \omega_{4}\right)
$$


has no resonances of order one or two:

$$
\Omega \cdot \nu \neq 0 \quad \text { for all } \nu \in \mathbb{Z}^{2} \quad \text { such that }|\nu|=1,2,
$$

where, for integer vectors, $|\nu|=\left|\nu_{1}\right|+\cdots+\left|\nu_{4}\right|$.

Remark: In the case of the ellipsoids of type $\mathrm{S}$, the eigenvalue problem for the matrix $X\left(b^{*}, M^{*}\right)$ factorizes into that for two $4 \times 4$ blocks. Since the construction of the matrix $T$ also factorizes, it is necessary to check the nonresonance condition (5.1) for all resonances of order one, but only for the four resonances $(1, \pm 1,0,0),(0,0,1, \pm 1)$ of order two.

For the construction of the Birkhoff normal forms we use the complex coordinates $U=$ $\left(W_{1}, W_{2}, W_{3}, W_{4}, Z_{1}, Z_{2}, Z_{3}, Z_{4}\right)$ defined by

$$
W_{j}=-\frac{i B_{j}+C_{j}}{\sqrt{2}}, \quad Z_{j}=\frac{B_{j}+i C_{j}}{\sqrt{2}}, \quad W_{j+2}=-\frac{i Q_{j}+P_{j}}{\sqrt{2}}, \quad Z_{j+2}=\frac{Q_{j}+i P_{j}}{\sqrt{2}} \quad(j=1,2)
$$

(note that $W$ are the coordinates, $Z$ the momenta). Since $\Xi=\Sigma U$, with

$$
\Sigma=\left(\begin{array}{cc}
\Sigma_{4} & 0 \\
0 & \Sigma_{4}
\end{array}\right) \quad \text { and } \quad \Sigma_{4}=\frac{1}{\sqrt{2}}\left(\begin{array}{cccc}
i & 0 & 1 & 0 \\
0 & i & 0 & 1 \\
-1 & 0 & -i & 0 \\
0 & -1 & 0 & -i
\end{array}\right)
$$

the Hamiltonian in terms of the complex coordinates $U$ is $H\left(U ; b^{*}, M^{*}\right):=\mathcal{H}\left(T \Sigma U ; b^{*}, M^{*}\right)$ and its quadratic part is

$$
H_{2}\left(U ; b^{*}, M^{*}\right)=\sum_{j=1}^{4} i \Omega_{j}\left(b^{*}, M^{*}\right) Z_{j} W_{j} .
$$

We now describe the construction of the Birkhoff normal form of order four of the Hamiltonian $H$. Given a function $f$ of $W=\left(W_{1}, W_{2}, W_{3}, W_{4}\right)$ and $Z=\left(Z_{1}, Z_{2}, Z_{3}, Z_{4}\right)$, we denote its Taylor series by $f(W, Z)=\sum_{j, k \in \mathbb{N}^{4}} f_{j k} W^{j} Z^{k}$, where $W^{j}=W_{1}^{j_{1}} W_{2}^{j_{2}} W_{3}^{j_{3}} W_{4}^{j_{4}}$ etc. For any integer vector $\nu \in \mathbb{Z}^{4}$, we define the $\nu$-th harmonic of $f$ by

$$
\langle f\rangle_{\nu}(W, Z)=\sum_{\substack{j, k \in \mathbb{N}^{4} \\ j-k=\nu}} f_{j k} W^{j} Z^{k} .
$$

The average of $f$ is its harmonic $\langle f\rangle_{0}$. The spectrum of $f$ is $\operatorname{Sp}(f)=\left\{\nu \in \mathbb{Z}^{4}:\langle f\rangle_{\nu} \neq 0\right\}$.

We construct the Birkhoff normal form by means of the so-called Lie method. Let $\Phi^{\chi}$ denote the time-one-map of the flow of the Hamiltonian vector field of a function $\chi$; the Lie transform generated by $\chi$ of a function $f$ is $f \circ \Phi^{\chi}$. If $f$ and $\chi$ are analytic in an open neighbourhood of a point (the equilibrium point, in our case), then $f \circ \Phi^{\chi}$ is also analytic in some sufficiently small, but nonempty, open neighbourhood of such a point and there one has $f \circ \Phi^{\chi}=\sum_{j=0}^{\infty} \frac{1}{j !} L_{\chi}^{j} f$. (We adopt the sign convention $L_{f} g=\{f, g\}=\frac{\partial f}{\partial Z} \frac{\partial g}{\partial W}-\frac{\partial g}{\partial Z} \frac{\partial f}{\partial W}$ for the Poisson brackets.)

The generating function $\chi$ of the Lie transform is constructed by solving the so-called homological equation, which has the form $\left\{H_{2}, \chi\right\}=f-\langle f\rangle_{0}$ for some function $f$. The solution of this equation is formally given by $\chi=\mathcal{S}_{\Omega}(f)$, where

$$
\mathcal{S}_{\Omega}(f)=\sum_{\nu \in \operatorname{Sp}(f) \backslash\{0\}} \frac{\langle f\rangle_{\nu}}{i \Omega \cdot \nu} .
$$


Obviously, this is well defined if $\Omega$ does not resonate with any $\nu \in \operatorname{Sp}(f)$, and if the series converges. In our case $f$ will always be a polynomial, either $f=H_{3}$ or $f=H_{4}^{\prime}$ (see below), so no convergence problems exist.

The fourth-order Birkhoff normal form of $H=H_{2}+H_{3}+H_{4}+\ldots$, if it exists, is constructed using two Lie transforms, which average the terms of degree three and four of $H$, respectively. The first Lie transform is generated by the solution

$$
\chi_{1}=\mathcal{S}_{\Omega}\left(H_{3}\right)
$$

of the homological equation $\left\{H_{2}, \chi_{1}\right\}=H_{3}$ and is well defined if

$$
\Omega\left(b^{*}, M^{*}\right) \cdot \nu \neq 0 \quad \forall \nu \in \operatorname{Sp}\left(H_{3}\right) .
$$

Since $\left\langle H_{3}\right\rangle_{0}=0$ and $L_{\chi_{1}}^{2} H_{2}=-L_{\chi_{1}} H_{3}$ one sees that this Lie transform conjugates $H=H_{2}+H_{3}+$ $H_{4}+\ldots$ to

$$
H^{\prime}=H_{2}+H_{4}^{\prime}+\ldots, \quad \text { where } \quad H_{4}^{\prime}=\frac{1}{2} L_{\chi_{1}} H_{3}+H_{4} .
$$

The second Lie transform is generated by the solution $\chi_{2}=\mathcal{S}_{\Omega}\left(H_{4}^{\prime}\right)$ of $\left\{H_{2}, \chi_{2}\right\}=H_{4}^{\prime}-\left\langle H_{4}^{\prime}\right\rangle_{0}$. It is well-defined if

$$
\Omega\left(b^{*}, M^{*}\right) \cdot \nu \neq 0 \quad \forall \nu \in \operatorname{Sp}\left(H_{4}^{\prime}\right) \backslash\{0\}
$$

and leads to the fourth-order Birkhoff normal form

$$
H^{\prime \prime}=H_{2}+H_{4}^{\prime \prime}+\ldots \quad \text { with } \quad H_{4}^{\prime \prime}=\left\langle\frac{1}{2} L_{\chi_{1}} H_{3}+H_{4}\right\rangle_{0} .
$$

For our purposes, the only important points are controlling the existence of the fourth-order Birkhoff normal form and testing that it possesses the convexity properties discussed above. Neither of these tasks require the actual computation of the generator $\chi_{2}$ : one needs only test (5.4) and compute $H_{4}^{\prime \prime}$.

B. Nekhoroshev stability of elliptic equilibria. Let us introduce the action functions $I=\left(I_{1}, \ldots, I_{4}\right), I_{j}=i W_{j} Z_{j}$, and regard $H_{4}^{\prime \prime}$ as a quadratic form on $\mathbb{R}^{4}$ by writing

$$
H_{4}^{\prime \prime}(I)=\frac{1}{2} I \cdot A\left(b^{*}, M^{*}\right) I
$$

where $A\left(b^{*}, M^{*}\right)$ is a $4 \times 4$ symmetric matrix. The Birkhoff normal form of order four (5.5) is said to be

- convex if the quadratic form $H_{4}^{\prime \prime}$ is definite, i.e. the eigenvalues of $A$ are either all positive or all negative.

- quasi-convex if the restriction of $H_{4}^{\prime \prime}$ to the subspace orthogonal to $\Omega$ is definite, i.e.

$$
\Omega \cdot I=0 \quad \text { and } \quad H_{4}^{\prime \prime}(I)=0 \quad \Longrightarrow \quad I=0 .
$$

- directionally quasi-convex if the restriction of the quadratic form $H_{4}^{\prime \prime}$ to the plane orthogonal to $\Omega$ is nonvanishing in the "first 16-ant", i.e.

$$
\Omega \cdot I=0, \quad H_{4}^{\prime \prime}(I)=0 \quad \text { and } \quad I_{1}, \ldots, I_{4} \geq 0 \quad \Longrightarrow \quad I=0
$$


Clearly, each notion generalizes the previous one.

If the Hamiltonian $H(W, Z)$ and its Birkhoff normal form of order four are analytic, then any of these conditions implies the Nekhoroshev-stability of the equilibrium, in the precise sense that for any small $\epsilon>0$ one has

$$
\|I(0)\| \leq \epsilon \quad \Longrightarrow \quad\|I(t)\| \leq \epsilon^{\alpha} \quad \text { for } \quad|t| \leq \exp \epsilon^{-\beta}
$$

for some positive constants $\alpha$ and $\beta$. There are different possible values for the constants $\alpha$ and $\beta$, which we report here with reference to the case under consideration of a system with four degrees of freedom:

- If the Birkhoff normal form is directionally quasi-convex, then one can take $\alpha=\beta=\frac{1}{4}$ and it is also possible to prove a stricter confinement of motions on correspondingly shorter times, namely $\alpha=\frac{1+k}{4+k}, \beta=\frac{1}{4+k}$ for any $k>0$ (see [10]).

- If the Birkhoff normal form is quasi-convex (directional quasi-convexity does not seem to be sufficient), then it is possible to prove the above estimates with $\alpha=1$ and $\beta=\frac{1}{16}$. (See [11].)

- If the Birkhoff normal form of order four is quasi-convex and it is possible to construct the Birkhoff normal form of order $s>4$, then one also has $\alpha=1, \beta=\frac{s-3}{16}$ (see [11]).

Remark: The original motivation for the quoted articles [10], [11] on the Nekhoroshev stability of elliptic equilibria was precisely the present study of the Riemann ellipsoids. Until a few years ago, all Nekhoroshev stability results assumed that the frequency vector would satisfy a strong nonresonance condition, typically a diophantine one. This would be insufficient for the study of a problem such as the present one, in which the frequencies of the equilibrium, and therefore its nonresonance properties, depend on continuously varying parameters. The notion of directional quasi-convexity was introduced in [2] in connection with the study of the Nekhoroshev-stability of the triangular points of the restricted three-body problem. As will be seen below, this notion also plays a central role in the study of the Riemann ellipsoids, since the majority of the ellipsoids are not quasi-convex. (Nekhoroshev estimates also hold if a sufficiently high order Birkhoff normal form exists and satisfies some "steepness" condition; directional quasi-convexity allows us to avoid these generalizations, the hypotheses of which would be rather difficult to verify and which would lead to significantly worse values for the constants $\alpha$ and $\beta$.)

C. KAM theory. If the Birkhoff normal form of order four is nondegenerate, in the sense that $\operatorname{det} A\left(b^{*}, M^{*}\right) \neq 0$, then KAM theory applies, ensuring that in any sufficiently small neighbourhood of the equilibrium, the majority of the initial data gives rise to motions which are quasi-periodic with four frequencies. (See e.g. [1] [16].)

\section{Nekhoroshev-stability of the Riemann ellipsoids: Numerical re- sults}

A. Results. We have numerically constructed the Birkhoff normal forms for a (quite large) number of sample Riemann ellipsoids. Specifically, within the existence region of each type of ellipsoid, we considered a mesh in the plane $\left(\frac{b_{2}}{b_{1}}, \frac{b_{3}}{b_{1}}\right)$ determined by vertical lines uniformly spaced at a distance .0025; the number of mesh points on each vertical lines typically varied between twenty and one hundred, depending on the length of the line (however, in some cases we used as many as five hundred points). This analysis leads to the following 

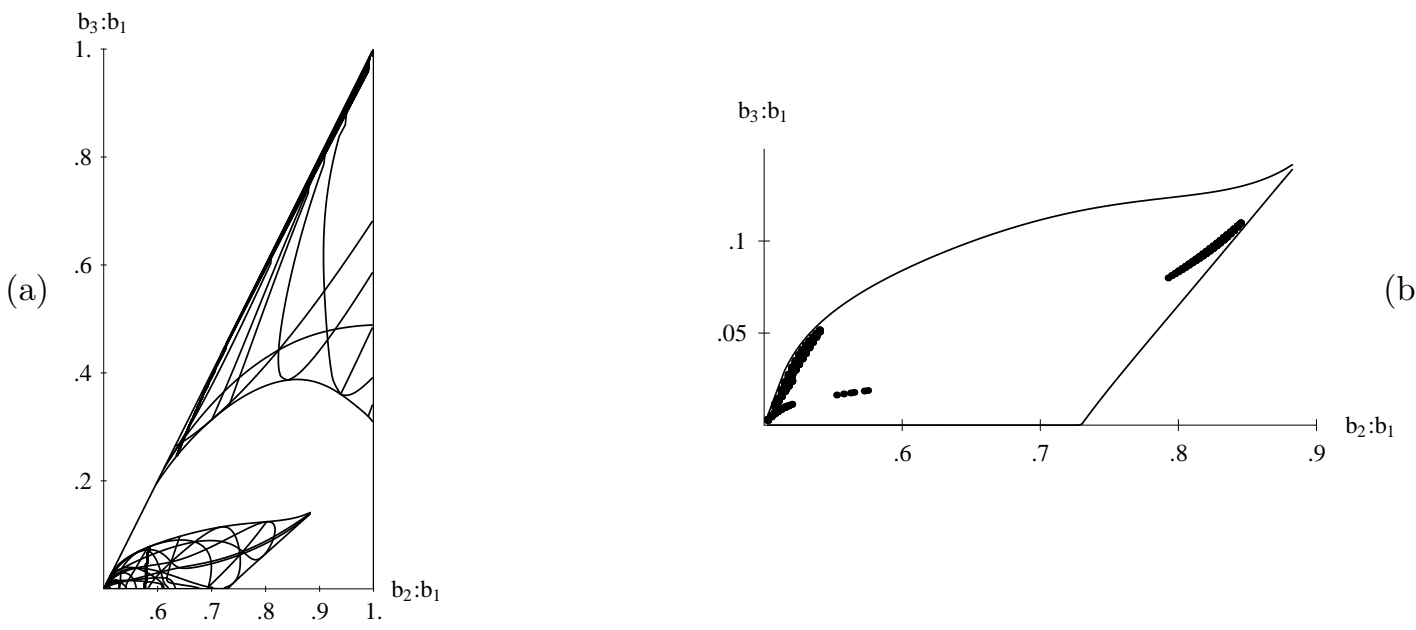

Figure 5: (a) The ellipsoids of type I that are resonant up to order four and (b) the quasi-convex Riemann Ellipsoids of type I in the lower region of ellipticity.

Numerical Conclusions 2. The Birkhoff normal form of order four exists and is directionally quasi-convex and nondegenerate for all the Riemann ellipsoids except for those lying on a finite number of curves in the set $\mathcal{B}$ corresponding to resonances of order up to four.

We regard the fact that all of the computed nonresonant Birkhoff normal forms are directionally quasi-convex as indicating that all nonresonant Riemann ellipsoids are Nekhoroshev stable. We investigated the existence of low-order resonances in greater detail, without restriction to the points of the considered mesh (see subsection 6.B for some details), finding that there are a finite number of curves in the plane $\left(\frac{b_{2}}{b_{1}}, \frac{b_{3}}{b_{1}}\right)$ at which at least one of the nonresonance conditions (5.1), (5.3), (5.4) is violated. Specifically, we found 8 different resonances for the type $\mathrm{S}_{2}, 52$ for the type I, 33 for the type II, and 47 for the type III. For example, figure 5 . a reports the resonant curves for the ellipsoids of type I.

We found that the Birkhoff normal form is quasi-convex only for a few ellipsoids of each type; in turn, very few of these ellipsoids are actually convex. For example, figure 5.b shows the quasiconvex ellipsoids in the lower region of elliptic type I ellipsoids. The determination of the quasiconvex ellipsoids has some interest because, as discussed in the previous Section, such equilibria may have stronger stability properties if they are nonresonant up to sufficiently high order. (From this perspective, the finer distinction between convexity and quasi-convexity does not instead seem to be as significant.)

Nondegeneracy of the Birkhoff normal forms implies that the KAM theorem applies, so that the majority of the motions near each equilibrium in the reduced phase space are quasi-periodic with four frequencies. "Reconstruction" of these motions then gives quasi-periodic motions of the Dirichlet problem, with up to (and in fact, typically) eight frequencies.

B. Numerical Procedures. We give now some information about the numerical procedures adopted here. The fourth-order Taylor expansion of the Hamiltonian in coordinates $(b, c, q, p)$, and hence the Hamiltonian matrix $X\left(b^{*}, M^{*}\right)$, has been constructed analytically for each type of 
ellipsoid. The coefficients of these polynomials have been evaluated for any ellipsoid in our mesh, and all other operations have then been performed on polynomials with numeric coefficients. These include:

- Computing the eigenvalues and the eigenvectors of $X\left(b^{*}, M^{*}\right)$ and testing for the presence of resonances of order one and two. Note that once the eigenvectors are known, the matrix $S\left(b^{*}, M^{*}\right)$ determining the coordinates $\Xi$ and $U$ is also known.

- Constructing the normal forms by means of formulas (5.5) and (5.2). This requires computing Poisson brackets, i.e. derivatives, and averages of polynomials with numeric coefficients; these are easily implemented operations.

- Checking the nonresonance conditions (5.3) and (5.4), which assure that the normal forms can be constructed. This involves determining the spectrum of a polynomial.

- Testing the normal forms, namely the numeric vector $\Omega$ and matrix $A$, for convexity, quasiconvexity, or directional quasi-convexity.

The chosen implementation of the latter two points requires some explanation.

Spectra and Resonances. We computed the spectra of $H_{3}$ and $H_{4}^{\prime \prime}$ at every point $\left(b^{*}, M^{*}\right)$ in the considered mesh and took the unions $\Sigma_{3}$ and $\Sigma_{4}$ of all these sets. (The union is taken because the numerically computed spectrum at a given point may lack very small harmonics.) We identified as resonances those $\nu \in \Sigma_{3} \cup \Sigma_{4}$ for which the scalar product $\Omega\left(b^{*}, M^{*}\right) \cdot \nu$ either vanishes or changes sign on our grid; of course, use of a finer grid could reveal additional resonances. (The resonant curves of figure 5 . a were constructed by computing the approximate zeros of $\Omega\left(b^{*}, M^{*}\right) \cdot \nu$, testing for changes of sign on a finer grid.)

Quasi and Directional Quasi-convexity. If the matrix $A$ is not convex, then the tests for quasiconvexity and directional quasi-convexity can be carried out as follows. Let $R \in \mathrm{SO}(3)$ be such that $R \Omega=(1,0,0,0)$, so that the Hessian of the restriction of the quadratic form $H_{4}^{\prime \prime}$ to the subspace orthogonal to $\Omega$ is the lower right hand $3 \times 3$ block $\tilde{A}$ of the matrix $R A R^{T}$. Quasi-convexity of $H_{4}^{\prime \prime}$ is equivalent to convexity of the matrix $\tilde{A}$.

Under the additional hypothesis that the restriction of $H_{4}^{\prime \prime}(I)$ to the subspace orthogonal to $\Omega$ is nondegenerate, 0 the test for directional quasi-convexity can be performed as follows. The quadratic form $H_{4}^{\prime \prime}$ is directionally quasi-convex iff any "asymptotic vector", namely any nonzero vector $I$ satisfying $I \cdot \tilde{A} I=0$, points out of the first 16 -ant, i.e. has at least one negative and one positive entry. Since these vectors form a cone (of dimension two, if $\tilde{A}$ is nondegenerate) we need only test the asymptotic vectors in the intersection of the cone and the unit sphere, namely two ellipses. In order to determine these ellipses, note that in the absence of quasi-convexity, the matrix $\tilde{A}$ has at least one eigenvalue of either sign. We may assume that the eigenvalues of $\tilde{A}$ satisfy $\alpha_{1}>0$ and $\alpha_{2}, \alpha_{3}<0$, replacing $\tilde{A}$ with $-\tilde{A}$ if necessary. (This is possible because $\tilde{A}$ and $-\tilde{A}$ have the same asymptotic vectors. Also, the nondegeneracy hypothesis assures that all of the eigenvalues are nonzero.) Let $\left\{\tilde{v}_{1}, \tilde{v}_{2}, \tilde{v}_{3}\right\}$ be an orthonormal eigenbasis associated to $\tilde{A}$, so that $S=\left(\tilde{v}_{1}, \tilde{v}_{2}, \tilde{v}_{3}\right) \in \mathrm{SO}(3)$ satisfies $S^{T} A S=\operatorname{diag}\left(\left|\alpha_{1}\right|,-\left|\alpha_{2}\right|,-\left|\alpha_{3}\right|\right)$. In this basis, the cone of asymptotic vectors is given by

$$
\left\{x=\left(x_{1}, x_{2}, x_{3}\right) \in \mathbb{R}^{3}: x_{1}^{2}=\frac{\left|\alpha_{2}\right|}{\alpha_{1}} x_{2}^{2}+\frac{\left|\alpha_{3}\right|}{\alpha_{1}} x_{3}^{2}\right\} .
$$

\footnotetext{
${ }^{6}$ This hypothesis is not crucial, but it is satisfied by all the computed ellipsoids.
} 
The two ellipses of unit vectors on this cone consist of the vectors

$\hat{x}^{ \pm}(\theta)=\left( \pm \sqrt{\frac{\left|\alpha_{2}\right|}{\alpha_{1}+\left|\alpha_{2}\right|} \cos ^{2} \theta+\frac{\left|\alpha_{3}\right|}{\alpha_{1}+\left|\alpha_{3}\right|} \sin ^{2} \theta}, \sqrt{\frac{\alpha_{1}}{\alpha_{1}+\left|\alpha_{2}\right|}} \cos \theta, \sqrt{\frac{\alpha_{1}}{\alpha_{1}+\left|\alpha_{3}\right|}} \sin \theta\right), \quad \theta \in S^{1}$.

In the original basis in the subspace orthogonal to $\Omega$, these vectors are

$$
\hat{I}^{ \pm}(\theta)=\left(\hat{I}_{1}, \hat{I}_{2}, \hat{I}_{3}\right)=S \hat{x}^{ \pm}(\theta) .
$$

These are three-dimensional vectors belonging to the subspace orthogonal to $(1,0,0,0)=R \Omega$, so the corresponding four-dimensional vectors are $I^{ \pm}(\theta)=R^{T}\left(0, \hat{I}_{1}, \hat{I}_{2}, \hat{I}_{3}\right)$. The normal form is directionally quasi-convex iff for every $\theta \in S^{1}$ the vector $I^{+}(\theta)$ has at least one negative and one positive entry. (It is obviously sufficient to consider only one of the two circles.) In the computations, we have tested this condition by checking that, at each of a number (typically 101) of uniformly spaced values of $\theta$ in the interval $0 \leq \theta \leq 2 \pi$, there are two eigenvalues whose product is negative.

\section{Conclusions}

In this work, we have posed the problem of the Nekhoroshev stability of those Riemann ellipsoids that are elliptic but of unknown Lyapunov stability. We have provided numerical evidence that all Riemann ellipsoids that are not resonant up to order four are Nekhoroshev stable. We have developed a consistent and rigorous Hamiltonian formulation of the problem on a four-to-one covering manifold, which validates the procedures (and clarifies the presence of a previously not understood discrete symmetry in the problem). Within this formulation we provided explicit, precise descriptions of the existence regions and of the momenta of the Riemann ellipsoids of the various types and we reviewed the existent results on the stability and ellipticity of the Riemann ellipsoids, showing (numerically) that some of the latter regions are significantly larger than was previously thought.

At the conclusion of this work, it is our opinion that several key features of the problem are not yet understood. Some open problems include:

- Properties of axisymmetric Riemann ellipsoids other than the Maclaurin spheroids.

- Analytic determination of the regions of ellipticity. Understanding of their subtle structure.

- Lyapunov stability or instability of those Riemann ellipsoids that are saddle points of the reduced Hamiltonian.

Of course, it would also be interesting to prove the Nekhoroshev-stability rigorously, that is, without recourse to numerical calculations, but this appears to be a formidable task at present.

In conclusion, we would like to mention a point of historic interest. Riemann's treatment of the Dirichlet problem is exceptionally advanced and uses several geometric constructions, including Poisson reduction, the amended potential, and the trivialization of the tangent and cotangent bundles of Lie groups, that are now considered to be central to modern geometric mechanics.

Riemann's apparent lack of concern with proving the actual instability of the saddle points of the reduced Hamiltonian is probably typical of that era - this error permeated the mechanical literature long after Lyapunov clarified the subject at the beginning of the twentieth century. At the dawn of a new century, the Lyapunov (in)stability of equilibria that are both elliptic and saddles of the 'obvious' Lyapunov function is still a formidable problem. Nekhoroshev stability provides a 
Fassò and Lewis: Riemann Ellipsoids

weaker, but very practical, notion of stability, which has the ultimate advantage of being compatible with Lyapunov instability.

\section{Appendix A: On the proof of Proposition 4}

The proof of Proposition 4, even though elementary, is too lengthy to be reported here. Hence we indicate the main steps in the form of Lemmas that we do not prove. The relative equilibria are the critical points of the reduced Hamiltonian $H_{\eta}: P_{\eta} \rightarrow \mathbb{R}$ and hence are given by the conditions

$$
\nabla_{b} H_{\eta}=0, \quad \nabla_{c} H_{\eta}=0, \quad m \times \nabla_{m} H_{\eta}=0 .
$$

Since $\nabla_{c} H_{\eta}=0$ iff $c=0$, we can equivalently search for critical points $(b, m)$ of

$$
\tilde{H}_{\eta}(b, m):=\frac{1}{2} m \cdot \mathbb{J}(b) m+\mathcal{V}(b) .
$$

Note that only the first equation in (8.1) depends on the potential $\mathcal{V}$.

Lemma 3 A nonzero vector $m=\left(m_{l}, m_{r}\right) \in\left(\mathbb{R}^{3}\right)^{2}$ satisfies $m \times \nabla_{m} \tilde{H}_{\eta}(b, m)=0$ iff either of the following alternative conditions is verified:

(i) $m_{l}$ and $m_{r}$ are both parallel to the same principal axis;

(ii) $m_{l}$ and $m_{r}$ both belong to the same principal plane, say $e_{i} \oplus e_{j}$ with $b_{i}>b_{j}$, but are not colinear with either principal axis, and, together with $b$, they satisfy any of the following three conditions (where $(i, j, k)$ is a permutation of $(1,2,3))$ :

(ii.1) $b_{j}<\left|b_{i}-2 b_{k}\right|$ and

$$
\begin{aligned}
\left(\frac{m_{l}^{i}-m_{r}^{i}}{m_{l}^{i}+m_{r}^{i}}\right)^{2} & =\left(\frac{b_{j}+b_{k}}{b_{j}-b_{k}}\right)^{4} \frac{b_{i}^{2}-\left(b_{j}-2 b_{k}\right)^{2}}{b_{i}^{2}-\left(b_{j}+2 b_{k}\right)^{2}} \\
\frac{m_{l}^{j}-m_{r}^{j}}{m_{l}^{j}+m_{r}^{j}} & =-\frac{m_{l}^{i}+m_{r}^{i}}{m_{l}^{i}-m_{r}^{i}}\left(\frac{b_{j}+b_{k}}{b_{j}-b_{k}}\right)^{2}\left(\frac{b_{i}+b_{k}}{b_{i}-b_{k}}\right)^{2} \frac{b_{i}+b_{j}-2 b_{k}}{b_{i}+b_{j}+2 b_{k}} .
\end{aligned}
$$

(ii.2) $b_{i}=2 b_{k}+b_{j}, m_{l}^{i}=-m_{r}^{i}$, and $m_{l}^{j}=m_{r}^{j}$.

(ii.3) $b_{i}=2 b_{k}-b_{j}$ and $m_{l}=m_{r}$.

Equations (8.2) and (8.3) imply that (8.2) is also valid with the index $i$ and $j$ exchanged. If either $m_{l}$ or $m_{r}$ vanish (the case of an irrotational ellipsoid), then equations (8.2) and (8.3) are equivalent to $k=2$ and $b_{1}^{2} b_{2}^{2}+b_{2}^{2} b_{3}^{2}+b_{1}^{2} b_{3}^{2}=3 b_{2}^{4}$.

We now consider the equation $\nabla_{b} H_{\eta}=0$. Set

$$
\tilde{\mathcal{B}}_{j}=\left\{b \in \mathcal{B}: G_{-}^{S}\left(b_{1}, b_{j}, b_{k}\right) \geq 0, G_{+}^{S}\left(b_{1}, b_{j}, b_{k}\right) \geq 0\right\}, \quad j=2,3
$$

where $(j, k)$ is any permutation of $(2,3)$ and

$$
\mathcal{B}_{i j}^{ \pm}=\left\{b \in \mathcal{B}: b_{j} \leq \pm\left(b_{i}-2 b_{k}\right), D\left(b_{i}, b_{j}, b_{k}\right) \neq 0, G_{\mp}^{R}\left(b_{i}, b_{j}, b_{k}\right)>0, G_{ \pm}^{R}\left(b_{j}, b_{i}, b_{k}\right)>0\right\}
$$

where $(i, j, k)$ is any permutation of $(1,2,3)$ with $i<j$. (We shall see below that some of these sets are empty.)

\footnotetext{
${ }^{7}$ By a permutation, we mean either an even or an odd permutation.
} 
Lemma 4 Assume $m=\left(m_{l}, m_{r}\right) \in\left(\mathbb{R}^{3}\right)^{2}$ is nonzero and satisfies $m \times \nabla_{m} \tilde{H}_{\eta}=0$. Then $\nabla_{b} \tilde{H}_{\eta}=0$ iff one of the following two conditions is satisfied:

(i) $b \in \tilde{\mathcal{B}}_{i j}, m_{l}=m_{l}^{j} e_{j}, m_{r}=m_{r}^{j} e_{j}$ and $\left(m_{l}^{j} \pm m_{r}^{j}\right)^{2}=G_{ \pm}^{S}\left(b_{1}, b_{j}, b_{k}\right)$ for either $j=2, k=3$ or $j=3, k=2$.

(ii) $b \in \mathcal{B}_{i j}^{-} \cup \mathcal{B}_{i j}^{+}, m_{l}=m_{l}^{i} e_{i}+m_{l}^{j} e_{j}, m_{r}=m_{r}^{i} e_{i}+m_{r}^{j} e_{j},\left(m_{l}^{i} \pm m_{r}^{i}\right)^{2}=G_{ \pm}^{R}\left(b_{i}, b_{j}, b_{k}\right),\left(m_{l}^{j} \pm m_{r}^{j}\right)^{2}=$ $G_{ \pm}^{R}\left(b_{j}, b_{i}, b_{k}\right)$ and, if $b_{i} \neq 2 b_{k} \pm b_{j}$,

$$
m_{l}^{j}-m_{r}^{j}=\operatorname{sign}\left(\left(2 b_{k}-b_{i}-b_{j}\right) \frac{\left(m_{l}^{j}+m_{r}^{j}\right)\left(m_{l}^{i}+m_{r}^{i}\right)}{m_{l}^{i}-m_{r}^{i}}\right) \sqrt{G_{-}^{R}\left(b_{j}, b_{i}, b_{k}\right)},
$$

for any permutation $(i, j, k)$ of $(1,2,3)$ with $i<j$.

The proof of part (i) of Proposition th is completed by showing that the "existence regions" of the previous Lemma coincide with those of the Proposition and that all the equilibria described in that Lemma are $\mathbb{Z}_{4} \times \mathbb{Z}_{2}$-equivalent or adjoint to those indicated in the Proposition:

Lemma 5 The sets $\mathcal{B}_{23}^{+}, \mathcal{B}_{23}^{-}$and $\mathcal{B}_{12}^{-}$are empty, while $\tilde{\mathcal{B}}_{2}=\mathcal{B}_{\mathrm{S}_{2}}, \tilde{\mathcal{B}}_{3}=\mathcal{B}_{\mathrm{S}_{3}}, \mathcal{B}_{13}^{-}=\mathcal{B}_{\mathrm{I}}, \mathcal{B}_{13}^{+}=\mathcal{B}_{\mathrm{II}}$, and $\mathcal{B}_{12}^{+}=\mathcal{B}_{\mathrm{III}}$.

Lemma 6 Under the assumptions of Lemma 勾, a point $(b, 0, m)$ is a relative equilibrium for some reduced system iff it is $\mathbb{Z}_{4} \times \mathbb{Z}_{2}$-equivalent to one of the points $\left(b, 0,\left(\mu_{\alpha}^{ \pm}(b), \mu_{\alpha}^{\mp}(b)\right)\right)$.

Part (ii) of the Proposition is a consequence of the following

Lemma 7 The maps $\mu_{\alpha}^{ \pm}, \alpha=\mathrm{S}_{3}$, II, III, are never zero. For $\alpha=\mathrm{S}_{2}$ and $\alpha=\mathrm{I}$, they vanish on the sets indicated in Proposition 国.

\section{Appendix B: On Chandrasekhar's linearization}

To facilitate the comparison of our results with Chandrasekhar's, in figure 6 we display the regions of elliptic ellipsoids of types II and III using the coordinates employed by Chandrasekhar, namely $\left(\frac{b_{1}}{b_{2}}, \frac{b_{3}}{b_{2}}\right)$ for the ellipsoids of type II and $\left(\frac{b_{1}}{b_{3}}, \frac{b_{2}}{b_{3}}\right)$ for the ellipsoids of type III. (For the latter, Chandrasekhar also uses the coordinates $\left(\frac{b_{2}}{b_{3}}, \frac{b_{2}}{b_{1}}\right)$; the corresponding figure should be compared to figure 3.b.) Among all these ellipsoids, Chandrasekhar identified as elliptic ("stable", in his terminology) only the ellipsoids in the lowest ellipticity region in figure 6.d.

As we have already remarked, Chandrasekhar's linearization of Riemann's equations yields the same results as ours (after correcting a few misprints that we report in the Remark below for the convenience of the interested reader). Hence, it seems probable that the meshes used by Chandrasekhar in his numerical study were too coarse, or too small, to capture all of the ellipticity regions shown in figure 6. Certainly, the very few ellipsoids whose frequencies are reported in [5] 


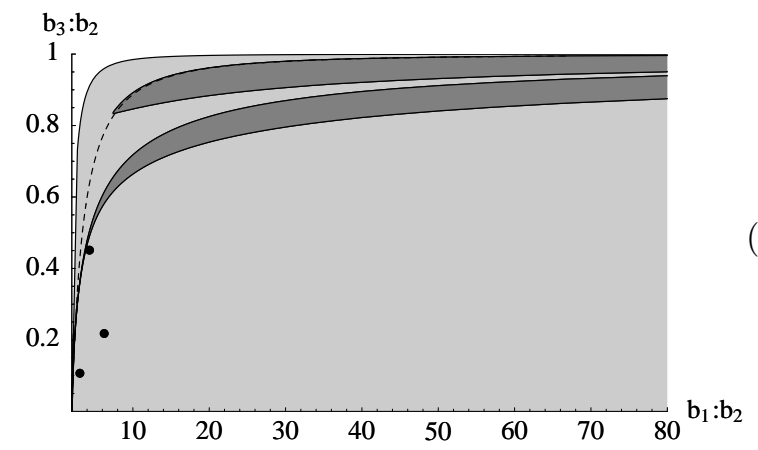

(a)
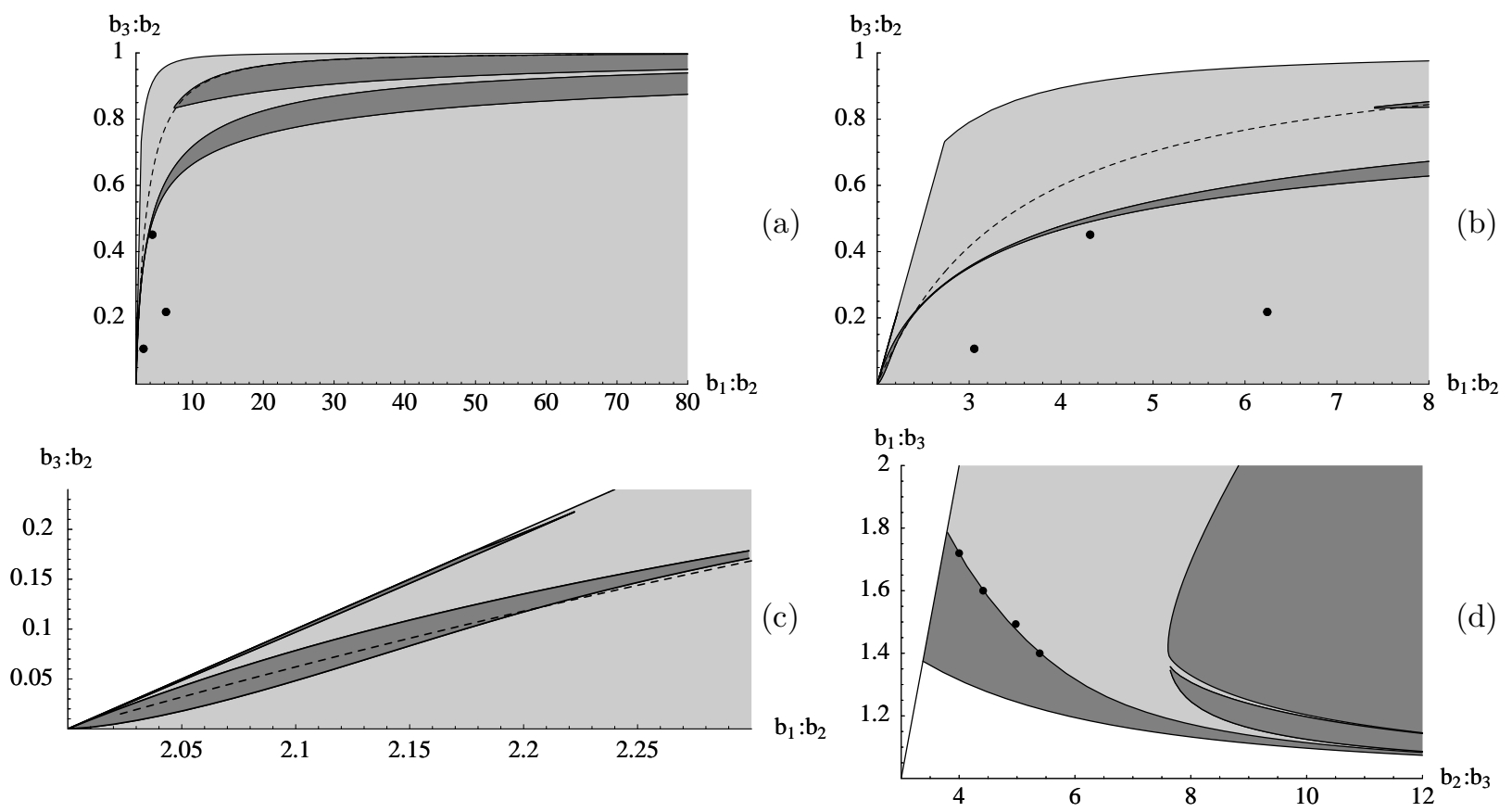

Figure 6: Regions of elliptic (dark gray) and non-elliptic (light gray) ellipsoids of types II (a-c), shown at three different resolutions, and III (d). The indicated individual points are the points tabulated in [5]. The dashed curve in $(\mathrm{a}-\mathrm{c})$ is the zero pressure curve.

(three of type II and four of type III, which are indicated by large points in figure 6) are consistent with our result.

Chandrasekhar did not exploit (nor, apparently, notice) the Lagrangian and Hamiltonian structure of the Dirichlet problem and worked with the full sixteen-dimensional Riemann's system, without exploiting the symmetry of the problem. The linearization of the reduced and unreduced systems are, of course, equivalent: the four additional frequencies appearing in the full system (two of which are identically zero) do not alter the ellipticity.

Remark: In [5], the frequencies of the linearized equations of motion are determined by the system of nine equations (116). The nine-by-nine determinant of the coefficients of this system is given in formula (133) of [5], where there are two misprints: the entry $(7,2)$ should be multiplied by $2+(\beta-2) \gamma a_{1}^{2} / a_{2}^{2}$, and the entry $(8,3)$ should be multiplied by $2+(\gamma-2) \beta a_{1}^{2} / a_{3}^{2}$. This determinant is reduced to the eight-by-eight determinant $(134)$, in which the entry $(1,8)$ should be changed to $\Omega_{2} \Omega_{3}+2 \frac{a_{1}}{a_{2}} \Omega_{2} \Omega_{3}^{\dagger}$, the entry $(2,7)$ to $\Omega_{2} \Omega_{3}+2 \frac{a_{1}}{a_{3}} \Omega_{2}^{\dagger} \Omega_{3}$ and the entry $(3,5)$ to $\Omega_{2} \Omega_{3}+\frac{a_{2}}{a_{3}} \Omega_{2}^{\dagger} \Omega_{3}^{\dagger}$; the entries $(8,1),(7,2)$ and $(5,3)$ should be changed accordingly: the entry $(i, j)$ is obtained from the entry $(j, i)$ by exchanging $\Omega_{k}$ and $\Omega_{k}^{\dagger}(k=2,3)$. Also, the symbol $B_{123}$ appearing in equation (135) of [5], should be defined as in equation (104), Chapter 3, of [6]. Let us explicitly note that these misprints are not the sources of the discrepancies between Chandrasekhar's and our conclusions: it is only after they have been corrected that equation (134) of [5] detects as elliptic the regions that Chandrasekhar describes as "stable". 


\section{References}

[1] V.I. Arnold, V.V. Kozlov and A.I. Neishtadt, Mathematical aspects of classical and celestial mechanics, in Dynamical system III, Volume 3 of Encyclopedia of Mathematical Sciences, V.I. Arnold editor (Springer-Verlag, Berlin, 1988)

[2] G. Benettin, F. Fassò and M. Guzzo, Nekhoroshev-stability of L4 and L5 in the spatial restricted threebody problem. Regular and Chaotic Dynamics 3, 56-72 (1998)

[3] P. Byrd and M. Friedman, Handbook of Elliptic Integrals for Engineers and Physicists (Springer Verlag, 1954)

[4] S. Chandrasekhar, The equilibrium and the stability of the Riemann ellipsoids. I. Astrophys. J. 145, 890-921 (1965)

[5] S. Chandrasekhar, The equilibrium and the stability of the Riemann ellipsoids. II. Astrophys. J. 145, 842-877 (1966)

[6] S. Chandrasekhar, Ellipsoidal figures of equilibrium (Yale University Press, New Haven, 1969)

[7] R. Cushman and L. Bates, Global aspects of classical integrable systems. (Birkhäuser,Basel, 1997)

[8] R. Dedekind, Zusatz zu der vorstehenden Abhandlung. J. Reine Angew. Math. 58, 217-228 (1860)

[9] G. L. Dirichlet, Untersuchung über ein Problem der Hydrodynamik. J. Reine Angew. Math. 58, 181$216(1860)$

[10] F. Fassò, M. Guzzo and G. Benettin, Nekhoroshev-stability of elliptic equilibria of Hamiltonian systems. Comm. Math. Phys. 197, 347-360 (1998)

[11] M. Guzzo, F. Fassò and G. Benettin, On the stability of elliptic equilibria. MPEJ - Mathematical Physics Electronic Journal 4, Paper 1 (1998)

[12] N. Lebovitz, On Riemann's criterion for the stability of liquid ellipsoids. Astrophys. J. 145, 878-885 (1966)

[13] D. Lewis, Lagrangian block diagonalization. J. Dyn. and Diff. Eqn. 4, 1-41 (1992)

[14] D. Lewis, Bifurcation of liquid drops. Nonlinearity 6, 491-522 (1993)

[15] P. Libermann and C.-M. Marle, Symplectic geometry and analytical mechanics. (D. Reidel, Dordrecht, 1987)

[16] K.R. Meyer and G.R. Hall, Introduction to Hamiltonian Dynamical Systems and the N-Body Problem (Springer, New York, 1991).

[17] L. Niederman, Nonlinear stability around an elliptic equilibrium point in a Hamiltonian system. Nonlinearity 11, 1465-1479 (1965)

[18] J.-P. Ortega and Ratiu, Stability of Hamiltonian relative equilibria. Nonlinearity 12, 693-720 (1999)

[19] G.W. Patrick, Relative equilibria in hamiltonian systems: The dynamic interpretation of nonlinear stability on the reduced phase space. J. Geom. Phys. 9, 111-119 (1992)

[20] B. Riemann, Ein Beitrag zu den Untersuchungen über die Bewegung eines flüssigen gleichartigen Ellipsoides. Abh. d. Köningl. Gesell. der Wiss. zur Göttingen 9, 3-36 (1860) 